\title{
Transcriptome-wide analysis of microRNA expression in the malaria mosquito Anopheles gambiae
}

\author{
Inna Biryukova ${ }^{1 *}$, Tao Ye ${ }^{2}$ and Elena Levashina ${ }^{{ }^{*}}$
}

\begin{abstract}
Background: microRNAs (miRNAs) are a highly abundant class of small noncoding regulatory RNAs that post-transcriptionally regulate gene expression in multicellular organisms. miRNAs are involved in a wide range of biological and physiological processes, including the regulation of host immune responses to microbial infections. Small-scale studies of miRNA expression in the malaria mosquito Anopheles gambiae have been reported, however no comprehensive analysis of miRNAs has been performed so far.

Results: Using small RNA sequencing, we characterized de novo A. gambiae miRNA repertoire expressed in adult sugar- and blood-fed females. We provided transcriptional evidences for 123 miRNAs, including 58 newly identified miRNAs. Out of the newly described miRNAs, 19 miRNAs are homologs to known miRNAs in other insect species and 17 miRNAs share sequence similarity restricted to the seed sequence. The remaining 21 novel miRNAs displayed no obvious sequence homology with known miRNAs. Detailed bioinformatics analysis of the mature miRNAs revealed a sequence variation occurring at their $5^{\prime}$-end and leading to functional seed shifting in more than $5 \%$ of miRNAs. We also detected significant sequence heterogeneity at the $3^{\prime}$-ends of the mature miRNAs, mostly due to imprecise processing and post-transcriptional modifications. Comparative analysis of arm-switching events revealed the existence of species-specific production of dominant mature miRNAs induced by blood feeding in mosquitoes. We also identified new conserved and fragmented miRNA clusters and A. gambiae-specific miRNA gene duplication. Using miRNA expression profiling, we identified the differentially expressed miRNAs at an early time point after regular blood feeding and after infection with the rodent malaria parasite Plasmodium berghei. Significant changes were detected in the expression levels of 4 miRNAs in blood-fed mosquitoes, whereas 6 miRNAs were significantly upregulated after P. berghei infection.

Conclusions: In the current study, we performed the first systematic analysis of miRNAs in A. gambiae. We provided new insights on mature miRNA sequence diversity and functional shifts in the mosquito miRNA evolution. We identified a set of the differentially expressed miRNAs that respond to normal and infectious blood meals. The extended set of Anopheles miRNAs and their isoforms provides a basis for further experimental studies of miRNA expression patterns and biological functions in A. gambiae.
\end{abstract}

\section{Background}

Hematophagous females of A. gambiae require animal blood for successful reproduction. Sequential blood intake is the main route for transmission of the protozoan parasite Plasmodium, the causative agent of malaria. The ability of Plasmodium parasites to establish infection in the vector

\footnotetext{
* Correspondence: biryukova@mpiib-berlin.mpg.de;

levashina@mpiib-berlin.mpg.de

${ }^{1}$ Department of Vector Biology, Max Planck Institute for Infection Biology, Berlin 10117, Germany

Full list of author information is available at the end of the article
}

mosquito can be compromised by many factors, including mosquito innate immune responses and factors derived from the blood of the human host [1,2]. A better understanding of regulatory circuits and mechanisms that regulate mosquito biology and contribute to vector resistance to Plasmodium parasites is urgently needed to curb malaria transmission.

miRNAs are approximately 22 nucleotides RNAs that regulate and influence a wide range of biological and physiological processes in metazoans and plants, playing 
instructive role throughout development and conferring robustness to gene expression $[3,4]$. In insects, the miRNA pathway has been best characterized in the fruit fly, D. melanogaster. The canonical miRNA biogenesis starts from transcription of endogenous primary miRNA transcripts typically produced by RNA polymerase II. The primary transcripts frequently contain multiple miRNA hairpin precursors, which are processed by the nuclear heterodimer DGCR-8 and the RNAse III enzyme, Drosha. The released $\sim 55-70$ nt cleavage product, called a premiRNA hairpin is exported to the cytoplasm. Once in the cytoplasm, the pre-miRNA is processed by another RNAse III enzyme, Dicer-1, yielding 22 nt small RNA duplexes [3,5]. In Drosophila, one of the strands (called the "guiding") is preferentially incorporated into an effector miRNA-induced silencing complex (miRISC) containing the Argonaute-family protein AGO1. The other ("passenger") strand is sorted into small interfering RNAs, siRISC complex with AGO2 effector protein [6]. A number of alternative Drosha/Dicer-independent pathways producing functional miRNAs have been reported [7]. However, regardless of the miRNA biogenesis diversity, the stability and silencing activity of mature miRNA predominantly require AGO effector proteins [7-9]. A comparative phylogenetic analysis of small regulatory RNA pathways revealed that major components of miRNA biogenesis and AGO effector proteins are conserved between A. gambiae and $D$. melanogaster [10]. It has been shown that siRNAmediated silencing in A. gambiae requires AGO2 [11], however no evidence of functional association between AGO1 and miRNAs in A. gambiae has been demonstrated so far.

miRNAs act as antisense guide for the miRISC complex to recognize target protein coding and non-coding RNAs. miRNA-target interactions are based on Watson-Crick base pairing between the miRNA seed region (nucleotides $2-8$ relative to its 5 -end) and target RNA $[3,12]$, typically leading to mRNA destabilization and translational repression [13]. miRNA expression levels and patterns rely on both steps of their biogenesis: transcription and processing, and are tightly regulated temporally and spatially during development $[14,15]$. The predominant mature miRNA can be produced from both the 5'- and 3'-arms of pre-miRNA hairpins. Selection of the functional arm, the precision of miRNA processing and post-transcriptional modifications play critical roles in the refining and diversifying of mature miRNA sequence and eventual functional activity $[16,17]$. Post-transcriptional modifications at the 3'-end, mostly non-template directed adenylation and uridylation, alter miRNA activity and stability by regulating either processing by Dicer-1 or incorporation into miRISC [18-21].

It has recently been reported that miRNAs can sense biotic stresses operating as an integral part of host immune responses to microbial infections, caused by viral, bacterial and Apicomplexan pathogens [22-24]. Interestingly, in $A$. gambiae the upstream control regions of the major components of miRNA biogenesis, Drosha, Dcr-1 and Ago-1 are enriched in the potential binding sites for NF-kappaBrelated transcription factors [10], presumably providing a link between the miRNA pathway and immune responses. Moreover, RNA silencing of Ago-1 and Dcr-1 in A. gambiae mosquitoes resulted in the increased survival of the rodent malaria parasite $P$. berghei [25]. Furthermore, a recent study using ribosome profiling in A. gambiae infected with the human malaria parasite $P$. falciparum revealed an enhanced association of $\sim 35$ mosquito immune-related transcripts, including two components of the miRNA pathway Dcr-1 and Drosha with polyribosomes [26].

In this study, using small RNA sequencing we performed the first systematic analysis of A. gambiae miRNAs in adult sugar- and blood-fed females. We provided transcriptional evidence for a wide diversity of mature miRNAs and their isoforms. Our analysis revealed significant sequence variations among mature miRNAs at their 3'-ends, mostly due to imprecise processing during their biogenesis and post-transcriptional modifications. Furthermore, our extended set of Anopheles miRNAs allows us to analyze their expression at an early time point after regular and infectious blood feeding. As a result, we identified 6 differentially expressed Anopheles miRNAs associated with the rodent malaria parasite $P$. berghei infections.

\section{Results}

\section{A. gambiae small RNA sequencing}

The majority of the originally annotated Anopheles miRNAs have been identified by sequence similarity with $D$. melanogaster miRNA orthologs using sequence and structure alignment $[27,28]$. In addition, a number of mosquito specific miRNAs identified in the closely related species, A. stephensi, Cx. quinquefasciatus and Ae. aegypti by cloning [29] and deep sequencing [30,31] has been shown to be conserved in A. gambiae. Among 67 Anopheles miRNAs reported in miRBase, less than a half were experimentally validated $[25,29,30]$. In order to identify and characterize de novo miRNAs in A. gambiae, we constructed small RNA libraries from sugar and blood-fed $A$. gambiae G3 adult females, including fecund females. Two independent libraries (biological replicates) were prepared and independently sequenced using the Illumina highthroughput sequencing platform, yielding a total of $\sim 67.1$ million sequence reads. We observed a significant correlation between two independent libraries sequencing results $\left(R^{2}=0.95\right.$ for sugar-fed and $R^{2}=0.96$ for blood-fed samples). Since the genome of the G3 strain has not been sequenced, the $A$. gambiae PEST strain genome was used 
as a reference. After adapter trimming and filtering out ambiguous reads, a total of $\sim 57.3$ million sequence reads were obtained and $\sim 46.2$ million sequence reads were aligned. Around $92.00 \%$ and $66.88 \%$ of the total reads were mapped to the reference genome in sugar and blood-fed mosquitoes, respectively (Figure 1A). Less than $1 \%$ of sequence reads were mapped to the $M$. musculus genome in sugar-fed mosquitoes, while in blood-fed samples $\sim 26.34 \%$ of sequence reads derived from mouse genome (Figure 1A). Analysis of the size distribution and abundance of all sequences within libraries between 17-30 nt revealed two major classes peaking at 21-23 nt and 25-28 nt (Figure 1B, C). Accordingly to the sequence analysis and genomic mapping, the first class of reads with a predominance of $22 \mathrm{nt}$ size was ascribed to miRNAs (Figure 1B, D). $36.71 \%$ and $25.21 \%$ of mapped reads were assigned to known miRNAs in sugar-fed females and blood-fed females, respectively (Figure 1D). In the second class, $26.02 \%$ and $36.00 \%$ of mapped reads were associated with repetitive elements in sugar and blood-fed mosquitoes, respectively (Figure 1D). Interestingly, the proportion of mapped reads derived from tRNAs and rRNAs was increased about 1.6-fold in blood-fed compared with sugar-fed mosquitoes (Figure 1D). This likely reflected the beginning of protein synthesis triggered by blood meal intake.

Using miRDeep2 pipeline, we provided transcriptional evidence based on the $5 \mathrm{p}$ - and $3 \mathrm{p}$-associated read abundance for 65 distinct Anopheles miRNAs previously reported in miRBase (Additional file 1: Figures S1-2). We identified bantam, miR-263a, miR-8, miR-10, miR-184 and miR-281 as the most abundantly expressed miRNAs in $A$. gambiae (Additional file 1: Figure S2). Among these miRNAs, only miR-184 has been previously characterized as the most frequently occurring miRNA in other mosquito species, Ae. aegypti, Cx. quinquefasciatus and Ae. albopictus [30,31]. Interestingly, a low number of reads supporting the $3 \mathrm{p}$-associated sequence and absence of the $5 \mathrm{p}$-associated reads were detected for the originally annotated by sequence similarity, mir-309 and mir-286 (Additional file 2: Table S1). The extremely low abundance of these miRNAs is probably due to their restricted spatial and temporal expression. Indeed, in Ae. aegypti orthologs of miR-309 and miR-286 were detected in embryos and not in the adult females [30]. Overall, these data support the authenticity of the originally annotated set of $A$. gambiae miRNAs in miRBase.

\section{A}
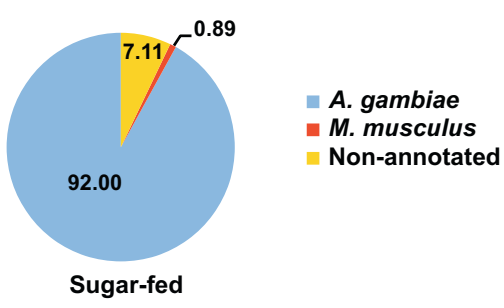

\section{B}

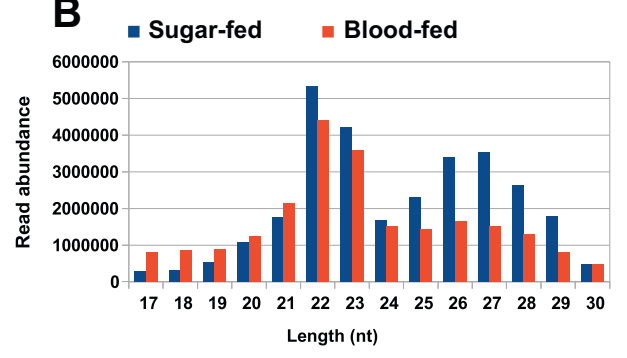

D

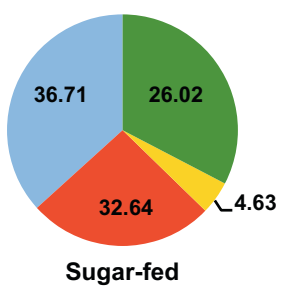

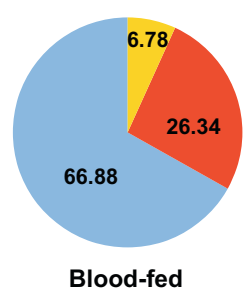

C
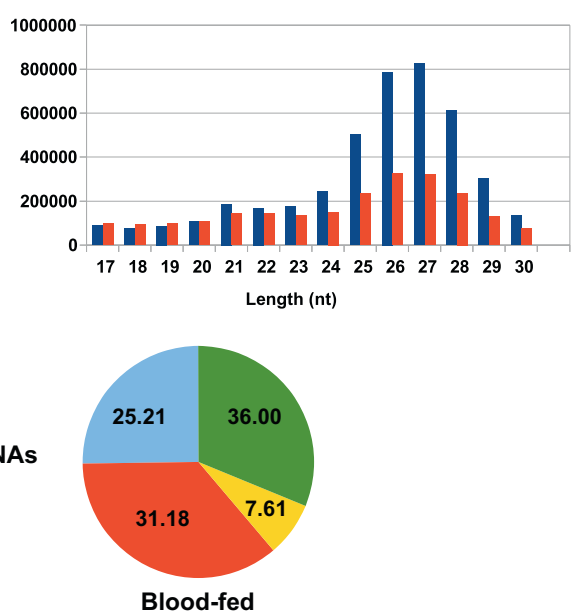

Figure 1 Sequencing summary of small RNAs in A. gambiae. (A) Read frequency for all sequences assigned to A. gambiae and M. musculus genomes in sugar and blood-fed mosquitoes. Size distribution for the total number of sequence reads (B) and for unique sequence reads (C). The abundance of the reads between 17 and $30 \mathrm{nt}$ from sugar and blood-fed mosquitoes are as indicated. (D) Read frequency for all sequences of small RNA reads. The identity and abundance of small RNA reads in sugar and blood-fed mosquitoes are as indicated. 


\section{Identification of novel A. gambiae miRNAs}

To detect putative A. gambiae miRNAs, we used miRDeep2 and a pipeline developed in-house. Only mapped sequence reads were used for the further prediction and expression analyses. We considered a general guideline for microRNA annotation in deep-sequencing data [32-34]. To refine results of the novel miRNA prediction, the following criteria were applied: i) detection of at least 10 sequence reads mapping to the miRNA hairpin per library; ii) valid miRNA precursors folding into stem-loop hairpins with folding energy less than $-15 \mathrm{kcal} / \mathrm{mol}$; iii) consistency of the 5 -end starting position, measured as an abundance of mapped sequence reads sharing the same 5'-terminus. Genuine miRNA loci produce so-called mature $(\mathrm{miR})$ and star $\left(\mathrm{miR}^{*}\right)$ sequences (originated from the preferred and non-preferred strands of miRNA duplex, respectively) that can be derived from both arms of the miRNA precursor. Hence, detection of miRNA sequence reads associated with the 5'- and 3'-arms of miRNA precursor increases a confidence in the novel miRNA annotation $[32,33,35]$. However, it has been reported that certain miRNA might lack miR* reads due to strong asymmetric strand selection during miRNA processing [34]. Therefore, in our study an absence of sequence reads supporting miR* was not considered as a decisive criterion for the annotation of low abundant novel miRNAs. To improve the accuracy of prediction of the novel miRNAs, only miRNAs identified at least in two independent cDNA libraries and in two biological experiments were included in the further analyses. Furthermore, potential novel miRNAs mapped to unknown contigs not associated with any of the three A. gambaie chromosomes, with multiple and inexactly mapped reads were filtered out.

In total, 123 distinct miRNAs were detected in the mosquito small RNA libraries (Additional file 2: Table S1), including distinct 65 known and 58 putative novel Anopheles miRNAs (Additional file 1: Figure S1 and Table 1, respectively). Sequence alignment revealed that 19 novel miRNAs shared a high level of sequence similarity with the miRNAs described in other insect species. Interestingly, although the Anopheles homolog of mir-71 was not predicted by miRDeep 2 in the original data set, the pipeline developed in-house revealed the corresponding $5 \mathrm{p}$ - and $3 \mathrm{p}$-associated sequence reads in our libraries (Table 1).

Evolutionary conservation of miRNA hairpin is considered as a reliable criterion for prediction of novel miRNA genes. Remarkably however, the degree of miRNA conservation does not always correlate with sequence read abundance [35]. For example, we observed that the nonpreferred strand of novel Anopheles mir-981 and mir-33 hairpins exhibit a high level of sequence similarity $(80 \%$ and $100 \%$, respectively) with known homologous hairpins. In contrast, the most abundant preferred strand of mir-33 exhibited sequence divergence in the seed region. Moreover, the preferred strand of mir-981 shows no significant sequence similarity with any known miRNAs. Since the preferred strand selection of certain miRNAs is not consistently associated with sequence conservation, our data demonstrate the limitations of miRNA prediction and annotation based on sequence similarity. We also detected 17 miRNAs with a sequence similarity restricted to the seed sequence only. Among those, putative miRNA orthologs were found for 4 Anopheles miRNAs in the closely related mosquito species Ae. aegypti and Cx. quinquefasciatus (Table 1; Additional file 1: Figure S3). Finally, the remaining 21 Anopheles miRNAs had no obvious sequence similarity with known miRNAs in other species (Table 1).

Closer examination of mature sequences derived from both arms of the predicted hairpins revealed that 20 novel candidate miRNA loci were associated with the presence of distal and proximal sequence reads. This might indicate that the candidate miRNA is a degradation intermediate [32]. Recently however, an example of a miRNA locus showing signatures of both, host mRNA degradation and miRNA processing via Drosha/Dicer cleavage has been reported in Drosophila [34]. 80\% of those Anopheles miRNAs mapped to intergenic regions. Yet, the possibility of dubious annotation of the predicted protein-coding genes and intergenic regions in Vector Base cannot be excluded. Manual inspection of the $\mathrm{miR} / \mathrm{miR}^{*}$ sequence reads comprising putative miRNA duplexes revealed that $70 \%$ of these miRNAs exhibit expected 1-2 nt 3'-end overhangs, the signature of substrates processed by the RNAse III enzymes, Drosha/Dicer. The remaining miRNA loci, including intron-derived (mirtrons) and intergenic miRNAs show unusual 3'-end overhangs. Among those, three intergenic-derived putative miRNAs (dme-mir-318, hsa-mir3605 and hsa-mir-183 seeds) exhibit very atypical 3'-end overhangs. Those miRNAs were considered to be lowconfidence candidate miRNAs (Table 1); and therefore further experimental studies will be required to demonstrate their functional association with miRISC.

\section{Validation of novel miRNA candidates}

Mature miRNA levels and stability require predominantly AGO effector proteins. Therefore, to increase the specificity of novel miRNA prediction, we analyzed miRNA levels in Ago1-silenced mosquitoes. As a control, we used mosquitoes depleted for AGO2, the effector of siRNAs. Small RNA libraries were prepared from mosquitoes injected with dsRNA against Ago1 and Ago2 and sequenced on the Illumina platform. Quantification of relative expression by qPCR showed that in the Ago1- and Ago2-silenced mosquitoes levels of Ago1 and Ago 2 were downregulated by $50 \%$ and $40 \%$, respectively (Figure 2A). Importantly, no cross-silencing of Ago1 
Table 1 Novel A. gambiae miRNAs

\begin{tabular}{|c|c|c|c|c|c|}
\hline miR sequence & Seed & $\begin{array}{l}\text { Predominant } \\
\text { arm usage }\end{array}$ & $\begin{array}{l}5 p-r a w \\
\text { read number }\end{array}$ & $\begin{array}{l}\text { 3p-raw read } \\
\text { number }\end{array}$ & Conservation \\
\hline GUCGACAGAGAGAUAAAUCACU & UCGACAG & $3 p$ & 5649 & 207160 & aae-miR-2940 \\
\hline UGUUAACUGUAAGACUGUGUCU & GUUAACU & $3 p$ & 17 & 76143 & aae-miR-999 \\
\hline CUAAGUACUAGUGCCGCAGGAG & UAAGUAC & $5 p$ & 63196 & 396 & aae-miR-252 \\
\hline UAGCACCAUUCGAAAUCAGUAC & AGCACCA & $3 p$ & 63 & 8597 & aae-miR-285 \\
\hline UCAAUUCCGUAGUGCAUUGCAGU & CAAUUCC & $5 p$ & 3981 & 39 & aae-miR-932 \\
\hline GUAGGCCGGCGGAAACUACUUGC & UAGGCCG & $3 p$ & 10 & 2313 & bmo-miR-2796 \\
\hline UGACUAGAGGCAGACUCGUUUG & GACUAGA & $3 p$ & 195 & 1637 & aae-miR-2945 \\
\hline UAGCACCAUGAGAUUCAGCUC & AGCACCA & $3 p$ & 86 & 1674 & aae-miR-998 \\
\hline UGACUAGACCGAACACUCGUAUC & GACUAGA & $3 p$ & 4 & 503 & aae-miR-286b \\
\hline UUGGUGUUAUAUCUUACAGUGAG & UGGUGUU & $3 p$ & 1 & 597 & dme-miR-971 \\
\hline GAAGGAACUUCUGCUGUGAUCU & AAGGAAC & $5 p$ & 142 & 7 & aae-miR-2944a \\
\hline GAAGGAACUCCCGGUGUGAUAUG & AAGGAAC & $5 p$ & 104 & 21 & aae-miR-2944b \\
\hline UAUCACAGCCAGCUUUGAAGA & AUCACAG & $3 p$ & 1858 & 25519 & aae-miR-2a \\
\hline UGGCAAGAUGUUGGCAUAGCU & GGCAAGA & $5 p$ & 24655 & 180 & aae-miR-31 \\
\hline AUUAGAAUGUGGAAUCUGUUUUU & UUAGAAU & $5 p$ & 1572 & 803 & $\begin{array}{l}\text { hsa-miR-561 seed, } \\
\text { conserved in aae, cqu }\end{array}$ \\
\hline UGAACACCCAUUUAUUGCCGACAGG & GAACACC & $3 p$ & 12 & 1341 & $\begin{array}{l}\text { miR conserved in aae, } \\
\text { miR-N3 }\end{array}$ \\
\hline CCCUGUGGAACACCAUGUACGAUGG & CCUGUGG & $3 p$ & 0 & 202 & $\begin{array}{l}\text { bmo-miR-3389 seed, } \\
\text { conserved in aae }\end{array}$ \\
\hline CAGUACUUCUGCAAUGCAACCC & AGUACUU & $3 p$ & 113 & 1577 & aae-miR-33 \\
\hline AUGGAUUCGAUCGAUCGAGUGC & UGGAUUC & 5 & 236 & 48 & dme-miR-976 seed \\
\hline UAGUACGAAUACGUACGAGGGA & AGUACGA & $3 p$ & 17 & 61 & aae-miR-2946 seed \\
\hline UGUGGUGGCACACUUUGACAAC & GUGGUGG & $3 p$ & 2 & 15 & tca-miR-3897 seed \\
\hline AAUGGCACUCUUGUUGGACAAG & AUGGCAC & $5 p$ & 24 & 2 & aae-miR-263a seed \\
\hline *CGAUACACGAACUGGGGCUCUCUCC & GAUACAC & $3 p$ & 49 & 139 & dme-miR-318 seed \\
\hline UUAUACUUCCUGCUUCACCGAU & UAUACUU & $3 p$ & 0 & 120 & aae-miR-305 seed \\
\hline GAUUUGUCCAAAAAGGAUG & AUUUGUC & $3 p$ & 0 & 14 & aae-miR-981 \\
\hline AGGAUUACGAUGAAGUGUUUGCGCC & GGAUUAC & $5 p$ & 135 & 0 & $\begin{array}{l}\text { bmo-miR-2846 seed, } \\
\text { conserved in aae }\end{array}$ \\
\hline AGAAAGACAUGGGUAGUGAGAU & GAAAGAC & $3 p$ & 1512 & 13384 & aae-mir-71 \\
\hline ACACGAUAAGAGGAAAGUUUACG & CACGAUA & $5 p$ & 79 & 11 & No, miR-N1a (Figure 5D) \\
\hline *UUGAAUUACGUCGGCAAUUUUUGGG & UGAAUUA & $3 p$ & 2 & 518 & hsa-miR-183 seed, miR-N4 \\
\hline UUAGAUUCCCAGAUCGUCAGAU & UAGAUUC & $3 p$ & 1 & 48 & hsa-miR-376a seed \\
\hline UAAGUGCAAAUCGUUGUAGUCGGUU & AAGUGCA & $5 p$ & 449 & 0 & $\begin{array}{l}\text { hsa-miR-519b seed, } \\
\text { miR-N6 }\end{array}$ \\
\hline UUGACUGUCGCCUCUGCGGAUG & UGACUGU & $5 p$ & 103 & 0 & hsa-miR-943 seed \\
\hline UUGGAGAUCAAAAGACGAUGUUUUU & UGGAGAU & $5 p$ & 20 & 2 & hsa-miR-1270 seed \\
\hline AUGGGGUUUGACCUGCUGGGC & UGGGGUU & $5 p$ & 41 & 0 & hsa-miR-3170 seed \\
\hline *UCUCCGUGGACGGCUGUCGAUGCC & CUCCGUG & $5 p$ & 148 & 1 & hsa-miR-3605 seed \\
\hline CGGGCUGUUGCAGCAGGUGCCU & GGGCUGU & $3 p$ & 0 & 67 & hsa-miR-4741 seed \\
\hline GUAGUCCGGAGUGGAGUC & UAGUCCG & $5 p$ & 30 & 0 & hsa-miR-6723 seed \\
\hline *UAGGCCCGACCAGAACUCGCUG & AGGCCCG & $3 p$ & 0 & 12 & hsa-miR-4747 seed \\
\hline AACGAGUUUCCCGAUACGACUG & ACGAGUU & $5 p$ & 282 & 4 & No \\
\hline CAUUACCGAUGGAUCCUUACCG & AUUACCG & $5 p$ & 187 & 28 & No, miR-N2 (Figure 5D) \\
\hline
\end{tabular}


Table 1 Novel A. gambiae miRNAs (Continued)

\begin{tabular}{llllll}
\hline UGCAUUCAGUGGGGCGGUCGU & GCAUUCA & $3 p$ & 1 & 103 & No \\
UAGACGAUUUCGGAAUGGCACAUCC & AGACGAU & $3 p$ & 5 & 492 & No \\
CCGGUGAACUGCUGUGCAGGGGCGC & CGGUGAA & $3 p$ & 3 & 209 & No \\
UCCGGCUACCGACUAACGGCUC & CCGGCUA & $3 p$ & 2 & 68 & No \\
ACUCCGGUCGACUCUGGACGAC & CUCCGGU & $3 p$ & 11 & 34 & No \\
UUGCGAGAGGACCUAUAAUGACU & UGCGAGA & $5 p$ & 35 & 0 & No \\
UUUUGGAACACAAGCUCGGCAGGCC & UUUGGAA & $3 p$ & 0 & 241 & No \\
AAUUGGACUCUAUAGCACCCU & AUUGGAC & $3 p$ & 3 & 78 & No \\
AACCGACAGAUCAUUGGCCAGA & ACCGACA & $3 p$ & 0 & 2243 & No \\
AGGAUUCGUAGUGCUACUGUGCAGA & GGAUUCG & $5 p$ & 249 & 1 & No \\
UACUUUCGCAAAUAGAUCGCUGCCU & ACUUUCG & $5 p$ & 617 & 2 & No, miR-N5 \\
UUGGUCUGAUUGCCUACACUGGCUU & UGGUCUG & $5 p$ & 497 & 4 & No \\
UAGGAUCUAUUGACAUUGCAGCCU & AGGAUCU & $5 p$ & 145 & 0 & No \\
CUCGCUGGCUGUCCGCAAACU & UCGCUGG & $3 p$ & 12 & 62 & No \\
UGAGAGAACGAAAGCAUUCCUU & GAGAGAA & $3 p$ & 1 & 34 & No \\
AAUUGGACUCUGUGGCACCCU & AUUGGAC & $5 p$ & 62 & 0 & No \\
CGCUCGACUAUUUAUCGCCCGAGA & GCUCGAC & $3 p$ & 2 & 252 & No \\
*ACGAGGCGAAGACUUUGUUGCC & CGAGGCG & $3 p$ & 2 & 12 & No \\
\hline
\end{tabular}

* low confidence candidate miRNA.

aae - Ae. aegypti.

cqu - C. quinquefasciatus.

dme $-D$. melanogaster.

bmo - B. mori.

hsa - H. sapiens.

tca - T. castaneum.

and Ago2 expression was detected suggesting that the observed silencing effect was specific to the corresponding target mRNA (Figure 2A). The TaqMan-based quantification and validation of the previously annotated miR-989 showed that the miR-989 expression levels were decreased by $65 \%$ in Ago1-silenced mosquitoes (Figure 2A). Accordingly to normalized RNA sequence read quantification in Ago-silenced libraries, miR-989 levels were decreased by $\sim 80 \%$ in Ago1-silenced mosquitoes, while in Ago2-silenced mosquitoes miR-989 levels have not been substantially changed (Figure 2A). The relative quantification of mature miR-989 in our small RNA libraries was consistent with qPCR measurements. We then investigated the effect of Ago1 and Ago2 silencing on the total miRNA expression levels in the dsRNA injected mosquitoes, using noninjected mosquitoes as a reference control (Figure 2B, C). We observed a $65 \%$ decrease in the total miRNA levels in Ago1-silenced mosquitoes, corresponding to a median $\log _{2}$ fold-change of -1.51 (Figure 2B). Silencing of Ago2 resulted in a $\log _{2}$ fold-change of 0.54 in miRNA expression levels (Figure 2C). In addition, as an internal negative control, we analyzed levels of small RNAs unrelated to the miRNA pathways. For snoRNAs, tRNAs and rRNAs, we detected 9\% decrease in Ago1-silenced mosquitoes (Figure 2D), that corresponds to the median $\log _{2}$ fold- change of -0.13, while in Ago2-silenced mosquitoes the median $\log _{2}$ fold-change of snoRNAs, tRNAs and rRNAs levels was 0.16 (Figure 2E).

We next examined individual changes in the expression levels of the newly identified miRNAs in Ago1- and Ago2-silenced libraries using expression of known Anopheles miRNAs as a positive control (Figure 2F). A small non-coding RNA not related to miRNAs U2 snRNA, invariantly expressed in all our libraries, was used to normalize miRNA expression levels. Similar $\log _{2}$ fold-changes $(-1.6)$ were observed for the expression levels of the newly identified miRNAs (miRDeep2) in Ago1-silenced mosquitoes as compared to the known miRNAs annotated in miRBase (-1.4) and Rfam (-1.3). In Ago2-silenced control, we did not observe substantial changes in the analyzed miRNA expression levels (Figure 2F). Furthermore, the expression levels of ncRNAs, unrelated to miRNAs (Rfam) were consistent in both Ago1- and Ago2-silenced libraries (Figure 2F). Remarkably, only one newly predicted miRNA (hsa-mir-4747 seed) with an extremely low number of reads showed no changes in expression levels in Agol-silenced mosquitoes, therefore, it was not considered as a confident miRNA candidate. Collectively, these data suggested that levels of mature Anopheles miRNAs were specifically affected by Agol silencing, thereby supporting 


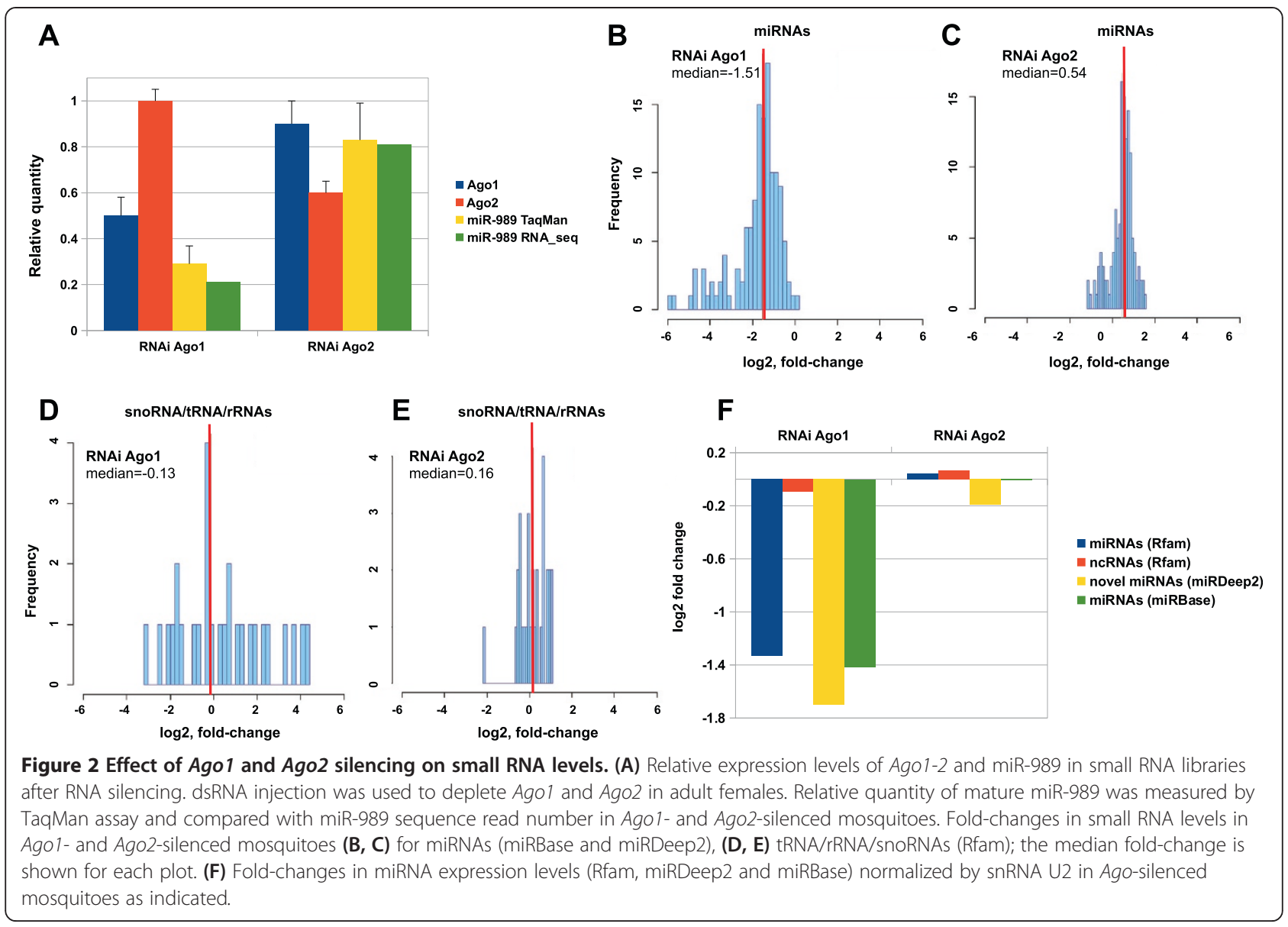

the accuracy of the miRNA prediction and annotation in this study.

\section{miRNA sequence heterogeneity}

Deep sequencing analyses revealed sequence heterogeneity at the 5'- and 3'-end of mature miRNA sequences, collectively called iso-miR variations. Such sequence variations can occur due to inaccurate processing by Drosha/Dicer-1, degradation and non-template sequence extension [19,20,34,36-41]. Analysis of sequence variations due to mismatches between the reads in our libraries and their corresponding genomic loci revealed $\sim 6.7 \%$ reads within mature miRNA sequences with 1 nt and more mismatching from their genomic loci. It has been reported that miRNA might be subjected to RNA editing by adenosine deaminase (A to $\mathrm{G}$ transition) and cytidine deaminase (C to $U$ transition) [34,38]. Therefore, we analyzed occurrences of the putatively edited mature miRNA sequence reads in our libraries. We did not find any evidence for the enrichment of $A$ to $G$ and $C$ to $U$ changes compared with other types of nucleotide alterations. Therefore, the observed sequence variations detected within mature miRNAs can be attributed to sequencing errors and/or to sequence variations between the query and the reference genome.

The specificity of target recognition is mostly determined by the 5 '-end of miRNAs [12]. Furthermore, the 5 -end precision and homogeneity of mature miRNAs show a high degree of evolutionary constraint. Therefore, we carefully analyzed cleavage accuracy of miRNA sequence reads associated with the 5'- and 3'-arms of miRNA precursors in our libraries. We detected high 5 -end fidelity of the $5 \mathrm{p}$ - and $3 \mathrm{p}$-associated sequence reads that was nearly identical (Figure 3A). Similar tendency was observed for the 5'-end homogeneity of mature and star miRNA sequences (Additional file 1: Figure S4). Importantly, we identified a group of miRNAs, including miR-283, miR-2, miR-210, miR-263a, miR-10 and miR252, with heterogenous 5'-ends (Figure 3C). mir-2-1 and mir-2-2 have been previously reported in miRBase as a miRNA with identical mature sequence, referred to as miR-2b and miR-2c, respectively. For miR-2, there were two abundant classes of the 5 -end, the one with the originally reported in miRBase and another, which was with 2 nt shorter (Figure 3C). In Drosophila, mir-2 subjected to alternative processing also produces two distinct miR-2 


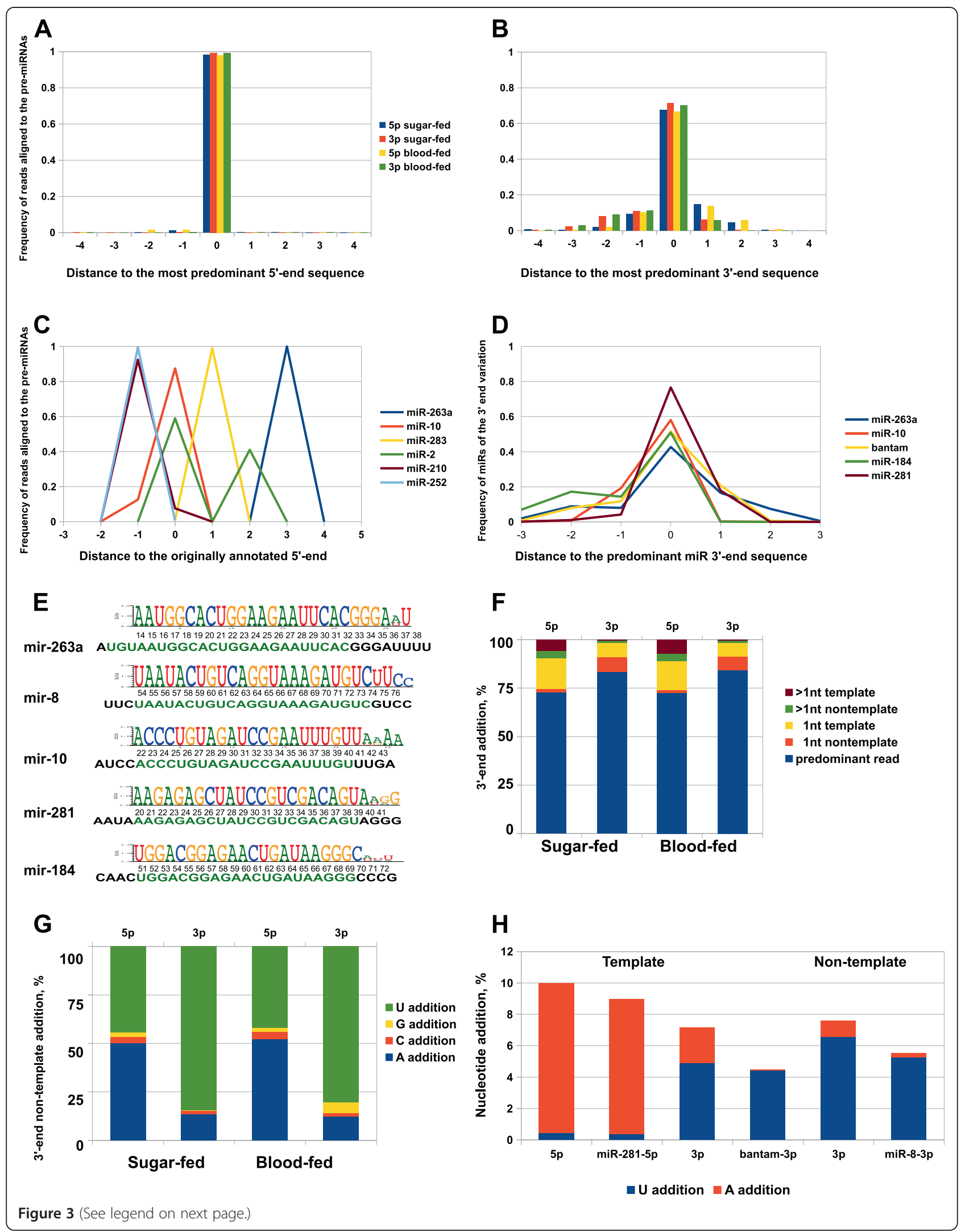


(See figure on previous page.)

Figure 3 miRNA sequence variation. (A-B) 5'- and $3^{\prime}$-end sequence heterogeneity of the $5 p$ - and $3 p$-derived reads as indicated. (C) Distribution of the predominant sequence reads grouped by their $5^{\prime}$-ends compared with the miRNAs reported in miRBase $v 19$. (D) Frequency of sequence reads grouped by their $3^{\prime}$-end compared to the predominant miRNA read. (E) Sequence compilations of five highly abundant mosquito miRNAs showing their mature sequence including the corresponding $3^{\prime}$-end sequence variations. Below, the mature miRNA sequence reported in miRBase is shown in green, adjacent genomic sequence is in black. (F-G) $3^{\prime}$-end extensions of the $5 p$ - and 3 p-sequences in sugar and blood-fed mosquitoes as indicated. $\mathbf{( H )}$ The percentage of $\mathrm{A}$ - and $\mathrm{U}$-tailed sequence reads of the extremely abundant miRNAs.

isoforms with 2 nt shifted 5'-ends with respect to each other [42]. We identified miR-10 and miR-210 with an extra 5' cytosine matched to the sequence of the premiRNA. For miR-10, two dominant mature species occur with the originally annotated 5 -end ( $\sim 87 \%$ of reads) and with an extra 5 cytosine ( $12 \%$ of reads) (Figure $3 C$ ). Similar variation at the 5 -end has been described in $D$. melanogaster miR-10 and miR-210, illustrating an example of the single hairpin generating mature miR with different abundant 5'-ends [34,37]. In Cx. quinquefasciatus, two dominant iso-miR species have been reported for miR210 , one of which contains an additonal cytosine nucleotide at its 5'-end [31]. Interestingly, A. gambiae miR-210 has only one dominant species with 5 '-end cytosine addition ( $~ 92 \%$ of reads). The originally annotated 5 '-end for miR-210 (miRBase) is represented by a small fraction of reads (7.6\%) in our libraries. Since in A. gambiae there are no paralogs of miR-10 and miR-210, this difference cannot be due to processing of mature miR from distinct homologous precursors. In Cx. quinquefasciatus, two dominant species have been reported for miR-252, one of which is 1 nt longer, with an extra cytosine residue at the 5'-end [31]. Similar modification at their 5'-end were detected in A. albopictus miR-252 (35\% of mature reads) [31]. For miR-252 identified in this study, we found $99 \%$ of mature reads with a template-directed cytosine addition at the 5'-end (Figure 3C). This consistent 5'-end cytosine addition had also been reported for Aedes miR-252 [30]. Interestingly, the predominant sequence of miR-263a was associated with a 3 -nt shifted 5 '-end; no sequence reads corresponding to the originally annotated sequence (miRBase v19) were detected in our libraries (Figure 3C, E). For miR-283, fewer than $1 \%$ of sequence reads corresponded to the mature sequence reported in miRBase v19. Instead, the majority of the annotated reads (more than 98\%) were $1 \mathrm{nt}$ shorter at their 5'-ends (Figure 3C). Collectively, the detected variations at the 5'-end of mature miRNAs that result in the functional-seed shifting, were observed for more than 5\% of Anopheles miRNAs across analyzed libraries.

Significant sequence heterogeneity was also observed at the 3'-end of miRNA sequence reads (Figure $3 \mathrm{~B}$ cf. 3A; Additional file 1: Figure S4). This is consistent with the general observation that 3 '-end sequence variation is more common than 5'-end variation [20,32,34,39]. Within all mature miRNA reads, around $14 \%$ reads have undergone more than 1 nt trimming or degradation at the 3'-end. Among the extremely abundant miRNAs, 1-2 nt trimming at the 3'-end was observed for $20 \%$ of reads from bantam and miR-10; for $17 \%$ of reads from miR-263a and for $32 \%$ of reads from miR-184 (Figure 3D). Next we extensively analyzed the 3 -end sequence extension (tailing) due to template and non-template directed nucleotide additions (Figure 3F, G). Analysis of the 3'-end composition revealed that the $5 \mathrm{p}$-associated sequence reads were preferentially subjected to template-directed extension of one or more nucleotides (Figure 3F). Furthermore, the frequency of non-template-directed additions was substantially lower at the 3'-end of 5p-associated sequences with respect to the 3p-associated reads (Figure 3F). Among the abundant Anopheles miRNAs, more than $60 \%$ of miR263a reads were $1-3$ nt longer at the 3 '-end than its mature sequence reported in miRBase (Figure 3D, E), which was represented by $\sim 2 \%$ of reads. Moreover, about one quarter of bantam and miR-281 reads was $1 \mathrm{nt}$ longer than their corresponding mature sequences reported in miRBase (Figure 3D, E). The described above nucleotide additions matched to the corresponding pre-miRNA sequences, indicating a template-directed origin of these additions most likely due to imprecise processing. In addition, we observed that miRNA loci, such as mir-277, let-7, mir-1174 and mir-279 produced from one fourth to one third of sequence reads tailed by template-directed adenine addition. Remarkably, a significant proportion of detected template-directed adenine and uracil additions in sequence reads derived from the $5^{\prime}$-arm is associated with miR-281 (Figure 3H). Moreover, the majority of templatedirected uracil extensions in the 3'-arm-derived reads are associated with bantam (Figure $3 \mathrm{H}$ ). It has been reported that sequence variation occurring due to imprecise cleavage by Drosha and Dicer are more frequent than nontemplate addition [39]. A similar tendency was observed for the 5p-associated sequence variations of Anopheles miRNAs (Figure 3F). However, the frequencies of template and non-template-directed additions were near similar at the 3'-end of the 3p-associated sequence reads (Figure 3F). The most predominant non-template directed nucleotide additions associated with the 5p-reads were adenine (44\%) and uracil (50\%) nucleotides (Figure 3G). We found that miR-125, miR-283, miR-10, miR-100, miR-281* and certain abundant isoforms of miR-279, miR-1174, miR-263a and miR-281 were substantially adenylated. Furthermore, 
we observed a bias toward uridylation in miRNA sequence reads (around $81-84 \%$ ) derived from the 3'-arm of the precursor miRNA across analyzed libraries (Figure 3G). Such modifications were mostly associated with miR-11, miR-14, miR-317, miR-277, miR-184, miR-8, miR-92b and miR-989. Strikingly, more than $80 \%$ of detected non-template directed uracil additions were associated with miR-8 (Figure 3G, E). The additions of cytosine and guanosine were detected only for $1-5 \%$ of sequence reads (Figure 3G). Taken together, the levels of the 3' uridylation and adenylation were substantially greater than other types of nucleotide additions in Anopheles miRNAs. This is consistent with the earlier reported observations for mammalian and insect miRNAs [19,34].

\section{Functional arm usage and shifts in sugar and blood-fed mosquitoes}

Analysis of the 5'- and 3'-arm usage in Drosophila revealed a slight bias towards 5'-arm usage (35). In contrast, in $A$. gambiae, we observed a bias towards 3'-arm usage (average $5 \mathrm{p} / 3 \mathrm{p}$ proportion was 0.4 ), which was consistent with abundance of sequence reads associated with the 3'-arm of precursor miRNAs (Figure 4A). It has been reported that selection and usage of the preferred arm can be dynamically regulated during development in a tissue-specific manner [20,32]. Switching in the functional arm usage changes the mature miRNA sequence selection and production, which consequently influences the target repertoire and function of a given miRNA. Notably, sequences associated with the non-preferred arm might also exhibit specific function in vivo [43]. To explore the preferred arm selection in Anopheles miRNAs, we reanalyzed arm usage reported in miRBase v19 and compared it with our data sets. We identified a group of miRNAs, mir-133, mir-1891, mir-278, mir-281, mir-965, mir-929 that produced corresponding $\mathrm{miR}^{*}$ at significantly higher levels compared to their mature miRs reported in miRBase (Table 2). Among these miRNAs, the mature miRNA sequence homologous to Anopheles miR281 \% has been reported earlier as predominantly expressed in Cx. quinquefasciatus [31]. In addition, the newly identified A. gambiae ortholog of mir-2940 also predominantly produced miR-2940* across all analyzed libraries (Table 1). Furthermore, we identified two Anopheles miRNAs, mir305 and mir-1889 that were not subjected to strong asymmetric strand selection (Table 2 ). They produced sequence reads associated with the 5'- and 3'-arm of the precursor at nearly the same levels, suggesting that preferred and non-preferred strands of the miRNA duplex are equally processed. The equal strand selection for mir-1889 has been previously reported in Ae. albopictus [31]. Interestingly, a shift from the predominant arm usage to a nearly equal $5 \mathrm{p} / 3 \mathrm{p}$ species production was observed for mir-219 and mir-3840 in the libraries derived from blood-fed
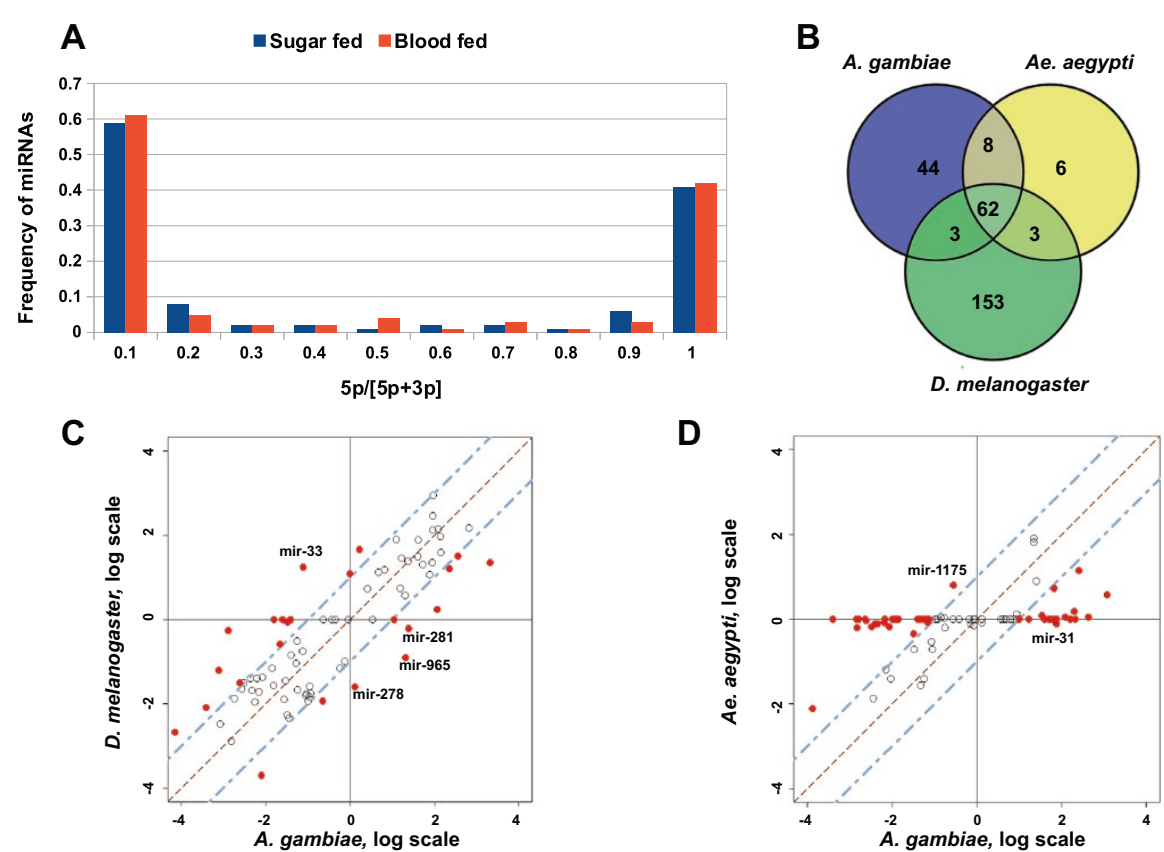

Figure 4 Arm usage analyses in Anopheles miRNAs. (A) Proportion of sequence reads associated with the $5^{\prime}$ arm of miRNAs with respect to the total number of reads in sugar and blood-fed A. gambiae mosquitoes. (B) Venn diagram showing the number of putative A. gambiae miRNA homologs in D. melanogaster and Ae. aegypti. (C-D) Comparison of relative arm usage between D. melanogaster and A. gambiae (C) and between two mosquito species, A. gambiae and Ae. aegypti (D). Shown are miRs exhibiting an arm usage bias; the dashed line indicates 10-fold differences in the relative arm usage. 
Table 2 Functional arm shifting in A. gambiae miRNAs

\begin{tabular}{|c|c|c|c|c|}
\hline \multirow[b]{2}{*}{ miRNA } & \multirow{2}{*}{$\begin{array}{c}\text { miRBase } \\
\text { annotated } \\
\text { predominant arm }\end{array}$} & \multicolumn{3}{|c|}{ RNA_seq } \\
\hline & & $\begin{array}{c}\text { detected } \\
\text { predominant arm }\end{array}$ & $\begin{array}{l}5 p / \\
3 p \_S F\end{array}$ & $\begin{array}{c}5 p / \\
3 p \_B F\end{array}$ \\
\hline miR-133 & $5 p$ & $3 p$ & 0.01 & 0.01 \\
\hline miR-1891 & $5 p$ & $3 p$ & 0.37 & 0.27 \\
\hline miR-278 & $3 p$ & $5 p$ & 1.33 & 1.34 \\
\hline miR-281 & $3 p$ & $5 p$ & 24.81 & 22.59 \\
\hline miR-965 & $3 p$ & $5 p$ & 23.56 & 9.56 \\
\hline miR-929 & $3 p$ & $5 p$ & 27.92 & 14.67 \\
\hline miR-1889 & $3 p$ & $5 p \sim 3 p$ & 0.93 & 0.72 \\
\hline miR-305 & $5 p$ & $5 p \sim 3 p$ & 1.02 & 0.85 \\
\hline miR-219 & $5 p$ & $5 p$ & 1.91 & 0.91 \\
\hline miR-3840 & novel miRNA & $3 p$ & 0.24 & 1 \\
\hline
\end{tabular}

SF-sugar-fed.

BF-blood fed.

mosquitoes (Table 2). However, overall blood meal intake did not affect the frequency of the relative arm usage more than 10-fold in A. gambiae libraries (not shown).

We next analyzed species-specific arm selection by comparing the relative arm usage between A. gambiae, $A e$. aegypti and D. melanogaster (miRNA data sets described in Methods). We retrieved insect miRNA homologs by considering 1:1 orthologous miRNA pairs between these species for the analysis (Figure 4B). Comparison of the relative arm usage revealed four miRNAs switching their preferred arms (Figure 4C). mir-965, mir-281 and mir-278 predominantly used the 5'-arm in Anopheles, whereas the 3'-arm was preferentially used in Drosophila. The opposite tendency was observed for mir-33. Comparison between sugar-fed Anopheles and Aedes female mosquitoes revealed no difference in the relative arm usage (not shown). Strikingly, 10-fold greater difference in relative arm usage was observed for mir-1175 and mir-31 orthologs in bloodfed Anopheles compared to Aedes females (Figure 4D). Taken together, our data revealed an existence of speciesspecific production of dominant mature miRNAs that might be involved in the regulation of the blood mealinduced physiological traits in the mosquito species and could be associated with the species diversification during Diptera evolution.

\section{Genomic organization, duplication and clustering of Anopheles miRNA genes}

Analysis of genomic organization revealed that around $68 \%$ of A. gambiae miRNAs were intergenic miRNAs. The remaining 32\% showed an overlap with the predicted transcripts annotated in Vector Base often mapping to the coding (sense) strand. Among those, $25 \%$ of miRNAs were located in introns, whereas $\sim 7 \%$ were mapped to exons. Expression of sense strand-derived miRNAs most likely coincides with expression of the host gene. Only three newly identified miRNAs were on the non-coding (antisense) strands of the overlapping transcripts. Such genomic organization of host genes and antisense miRNAs might have a regulatory function to interfere with miRNA transcription, or might affect the host gene to influence mRNA splicing or to target sense mRNA. Interestingly, known insect-specific antisense transcribed miRNAs mir307 and $i a b-4$ do not exhibit conserved genomic organization in A. gambiae. Furthermore, we did not find any examples of miRNAs convergently transcribed from both sense and antisense strands in A. gambiae.

miRNA gene duplication is an important source of phenotypic plasticity, robustness and diversity in development. The previously described sets of Anopheles miRNA paralogous genes include mir-375, mir-965, mir2, mir-92, mir-9 and mir-263 (miRBase v19). We analyzed multiple mapped $A$. gambiae miRNAs, scoring the new paralogous miRNAs as a duplication of pre-miRNA precursors and/or mature and star sequences. miRNAs detected in unknown contigs not associated with any of three A. gambaie chromosomes, with mismatches to the reference genome were filtered out. We further extended paralogous miRNA gene sets by describing new homologs of known Anopheles miRNAs and of new miRNAs annotated in this study (Table 1; Additional file 1: Figures S3; S5). Novel mir-276 (mir-276-2) shares 100\% similarity with the mature and star sequences of the originally annotated mir-276 (miRBase), showing sequence divergence only within the terminal loop. Mature miR-309 sequence was represented by two perfect copies, whereas star and terminal loop sequences of the two mir-309 paralogous genes were not conserved. Moreover, mir-2944 identified in this study was represented by three homologous genes: mir-2944a-1/-2 and mir-2944b (Table 1). Finally, we annotated a novel member of the mir-2 gene family $($ mir $-2 b+c)$, mir-2a (Table 1, Figure 5C).

miRNAs are frequently clustered in the genome (miRBase) and are most likely expressed from polycistronic transcripts. We analyzed the organization of miRNA clusters and their evolutionary conservation within the mosquito species (Figure 5A). The analysis of miRNA clusters in A. gambiae genome revealed that approximately $40 \%$ of miRNAs are clustered on the X chromosome. Around $36 \%$ of miRNAs were closely linked on the chromosomes 2 and 3R, however no miRNA clusters were found on the chromosome $3 \mathrm{~L}$. We observed that $20 \%$ of Anopheles miRNAs were closely linked within $1 \mathrm{~kb}$ genomic distance. For larger clusters, around 30\% of miRNAs were linked within 5-10 kb genomic distance and approximately $40 \%$ of miRNAs were closely linked at the distance of $50 \mathrm{~kb}$ and less (Figure 5A). The mean number of miRNAs per cluster was two for $1 \mathrm{~kb}$ genomic distance and three miRNAs for 5 - $50 \mathrm{~kb}$ genomic distance (Figure 5A). Collectively, these data indicates that 


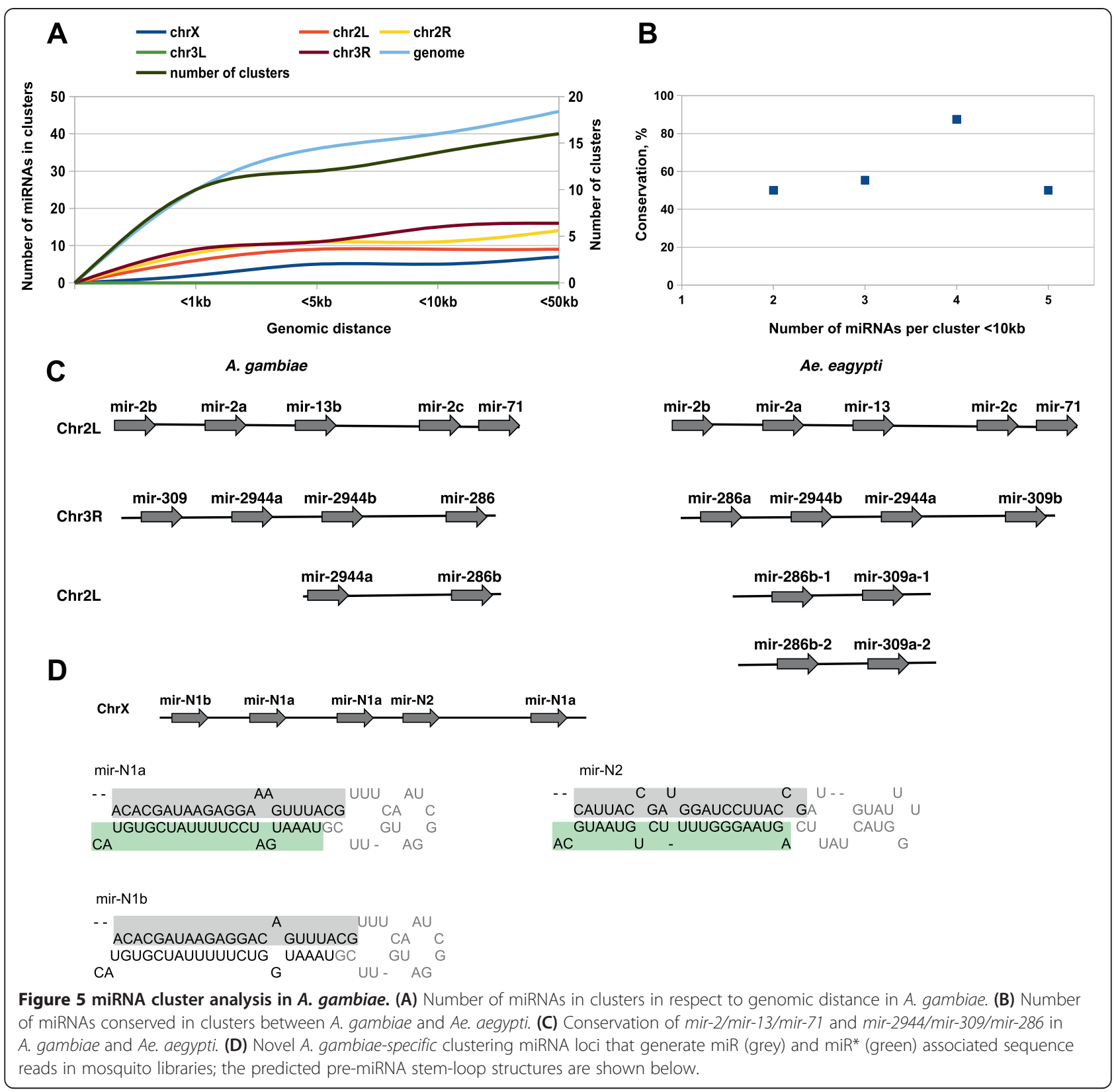

one third of Anopheles miRNAs are closely linked within $50 \mathrm{~kb}$. We also observed that all closely linked Anopheles miRNAs are located on the same stand within analyzed clusters. Expression levels of clustered miRNAs were significantly correlated across all mosquito libraries $\left(R^{2}=\right.$ 0.99). Nevertheless, further regulation at the processing level might provide the eventual "activity" patterns distinct from the expression patterns for the neighboring miRNAs of the same cluster. The proportion of clustered miRNAs producing the dominant miRNA from the same arm was 0.85 within a distance of $50 \mathrm{~kb}$ and less, and 0.57 for nonclustered miRNAs scored at a distance more than $50 \mathrm{~kb}$, revealing a strong bias towards the same arm selection for clustered miRNAs with respect to non-clustered miRNAs.

We further analyzed a conservation of clustered miRNAs between Anopheles and Aedes genomes within $10 \mathrm{~kb}$ of genomic distance (Figure 5B). Since the repertoire of miRNAs largely overlaps between these mosquito species, comparison of miRNA clusters consistently revealed a high level of conservation between A. gambiae and Ae. aegypti. Around $68 \%$ of clusters containing four miRNAs maintained their closely linked organization in both species. Moreover, more than $75 \%$ of conserved orthologous miRNAs grouped in clusters of two, three and five miRNAs 
were linked in Aedes. Importantly, this analysis allowed us to revise the clustered miRNA sets in Anopheles. We further extended previously predicted by sequence similarity mir-2/mir-13 cluster (miRBase v19) by providing evidences for mir-2a and mir-71 expression in our study (Figure 5C). Notably, the organization of mir-2/mir-13/ mir-71 cluster is highly conserved in invertebrates [35]. Other examples are the newly described mir-2944a and mir-2944b, which formed a cluster with the previously annotated mir-309 and mir-286 (Figure 5C). Interestingly, we identified a fragmented duplication of the mir-2944/ mir-309/mir-286 cluster, which included only mir-2944a/ mir-286 and lacked mir-309. In Ae. aegypti, the fragmented cluster contains only mir-286 and mir-309, lacking mir-2944. Although clustering of mir-2944/mir-309/mir286 was conserved between A. gambiae and Ae. aegypti, the fragmented clusters described above represent an example of miRNA cluster diversification between these mosquito species. Anopheles mir-285, identified in this study, is closely linked with mir-11 at a distance shorter than $1 \mathrm{~kb}$. However, the clustering organization of these miRNAs is not conserved in Aedes. In A. gambiae, mir965 was represented by a cluster of two paralogous genes, mir-965-1 and mir-965-2, whereas only a single mir-965 ortholog was described in Aedes. Finally, we identified a novel cluster containing two A. gambiae-specific mir-N1 and mir-N2 that shared no significant homology with any known miRNAs (Figure 5D). The miRNA expression profiling revealed a relatively low abundance of these miRNAs (Table 1). miR-N1 might potentially arise from four clustered hairpin precursors. Three out of four hairpin precursors represented a perfect duplication of mir-N1. The fourth hairpin precursor was slightly divergent showing minor sequence changes in the mature and star sequences supported by sequence reads in our libraries. In summary, miRNA clustering is highly conserved between Aedes and Anopheles indicating the orthologous origin of these clusters. The described A. gambiae-specific miRNA gene clustering and cluster fragmentation are most likely an example of the evolutionary "young" species-specific miRNA segregation emerging through gene duplication followed by sequence divergence due to mutational drift.

\section{Regulation of miRNA expression by regular and infectious blood feeding}

In order to identify miRNAs regulated by blood feeding, we first compared the relative abundance of miRNAs in sugar-fed mosquitoes and in mosquitoes $3 \mathrm{~h}$ after a feeding on a mouse (Figure 4A). Using $\log _{2}$ fold-change, more than 1.5 as a threshold, we revealed changes in abundance of miR-7, miR-92a, miR-317 and newly described miR-N3 (Figure 6A). The expression levels of abundant miR-7 and miR-92a were more than 5- and 25-fold upregulated by blood feeding, respectively. miR-92a is a highly conserved miRNA in animals, including the mammalian blood-hosts of mosquitoes, with minor species-specific sequence variations at the 3' end (Figure 6B). Therefore, we examined the origin of miR-92a elevated levels in the analyzed libraries. The analysis of miR-92a-associated sequence reads in sugar-fed mosquitoes revealed that miR-92a was represented by two classes: a $22 \mathrm{nt}$-long mature sequence reported in miRBase as aga-miR-92a and another sequence of a $20 \mathrm{nt}$ (Figure 6B, C). In blood-fed females, four distinct predominant miR-92a classes were detected, including the above described Anopheles-specific mature sequences and two mature sequences assigned to M. musculus (miRBase v19). Around $90 \%$ of the total mature miR-92a reads in blood-fed mosquitoes were associated with the two isoforms of mmu-miR-92a (Figure 6B, C). Interestingly, the predominant mouse mmu-miR-92a sequence isoform detected in the blood-fed libraries was identical with the human hsa-miR-92a sequence reported in miRBase (Figure 6B). No significant changes were observed in the levels of endogenous Anopheles agamiR-92a (Figure 6C). Taken together our data suggest that the dramatic change in miR-92a levels resulted from the exogenous miRNA intake occurred during blood feeding.

To assess the early responses of Anopheles miRNAs specific to $P$. berghei infection, we compared miRNA expression levels in females $3 \mathrm{~h}$ after regular and infectious blood feeding. We identified 6 miRNAs responding to the P. berghei parasite presence (Figure 6D). Among those, two highly expressed miRNAs, miR-317 and miR2940 were more than 5 - and 3 -fold upregulated by P. berghei infection, respectively. The remaining differentially expressed miRNAs showed a relatively low abundance. Overall, the Anopheles miRNAs identified here might represent early and dynamically regulated mosquito sensors that respond to normal and infectious blood meals.

\section{Discussion}

In the current study, using small RNA sequencing, we performed the first systematic analysis of Anopheles miRNAs and iso-miRs. Highly expressed Anopheles miRNAs (e.g. bantam or miR-263a) are represented by numerous mature sequence variants that vary in their abundance across all analyzed libraries. We characterized 5 '-end variations in detail, which alter miRNA seed sequences, thereby diversifying the target specificity of a given miRNA. We showed that more than 5\% of Anopheles miRNAs exhibit shifts in the functional seed sequences. These include newly described predominant isoforms of known Anopheles miRNAs, such as miR-263a, miR-283, or miR-210 that are abundantly expressed across all libraries. The detected here predominant mature sequences of these miRNAs are distinct from those originally predicted by sequence similarity (miRBase). Interestingly, Anopheles 


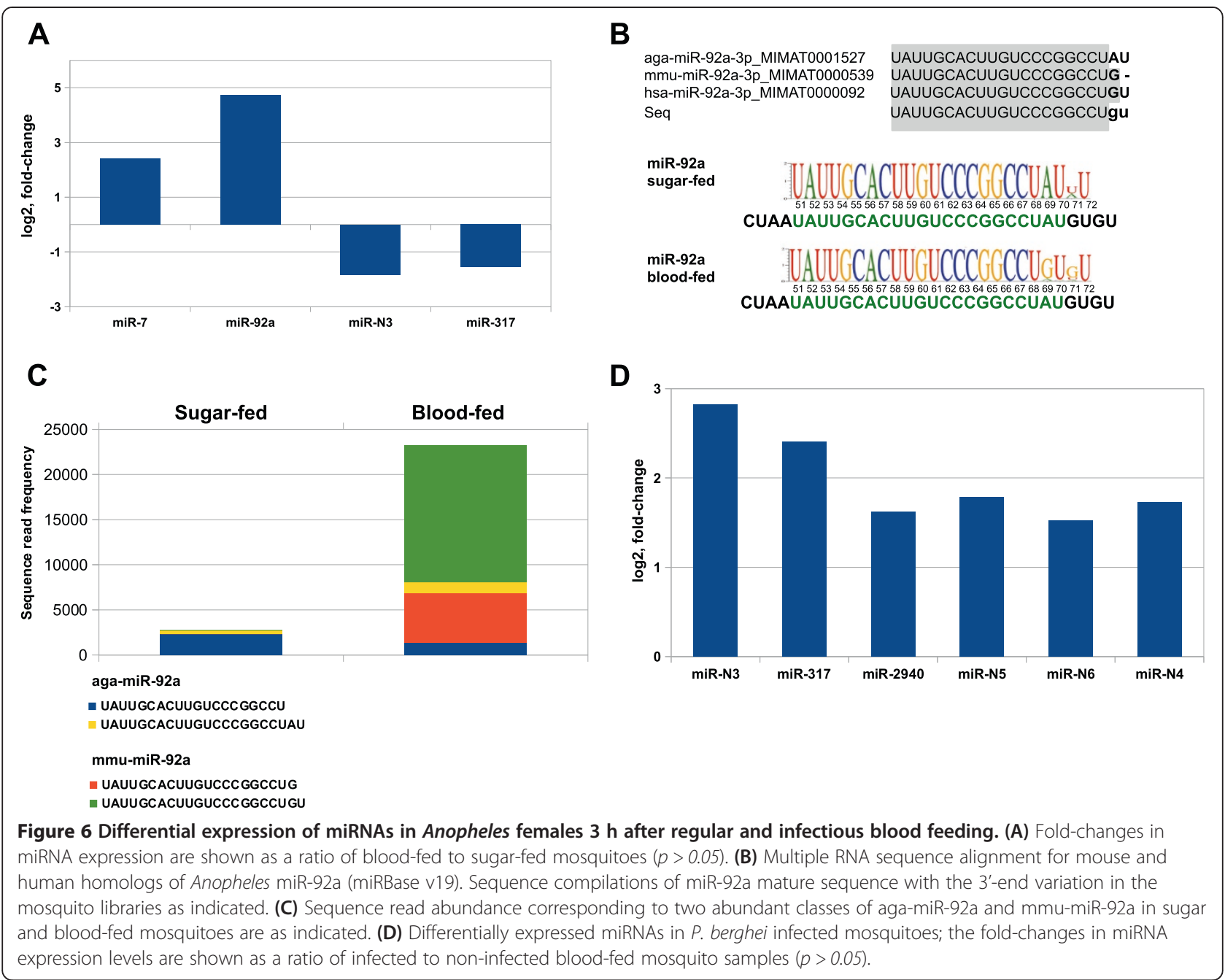

miRNA loci such as mir-2b/-c are capable of producing equal numbers of the highly expressed iso-miR species with alternative 5'-ends. Remarkably, Drosophila homologous mir-2 is also subject to alternative Dicer processing [42]. The origin of the conserved processing pattern of mir-2 homologs is unclear. It has been reported recently, that the alternative length of mature miRNAs can be regulated by the interacting partners of RNAse III enzymes [44]. Therefore, generation of alternative iso-miR-2 may require specific trans-acting factors regulating precision of Drosha/Dicer cleavage.

We also laboriously characterized miRNA sequence heterogeneity at 3'-ends (Figure 3, Additional file 1: Figure S4). The substantial fraction of Anopheles miRNAs exhibits 3 '-end trimming and additions. Among nongenomematching (non-template-directed) 3'-nucleotide extensions, uracil and adenine additions were the most predominant modifications of the mature miRNA sequences across analyzed mosquito libraries. Strikingly, in A. gambiae, $80 \%$ of nontemplate-directed urydilated reads derived from
miR-8. The number of described 3' additions in this study might be significantly underestimated since certain adenine and uracil additions map to adjacent genomic sequence of the pre-miRNA (e.g. miR-281, let-7 or bantam) and therefore, it was not possible to discern unambiguously between template- and nontemplate-directed additions. It has been reported that 3' adenylation stabilizes miRNAs $[40,45]$, whereas uridylation targets miRNAs for degradation $(21,41)$. In other studies, a bias towards 3' uridylation was observed for the AGO-immunoprecipitated miRNA fraction [20]. In contrast, it has been shown that 3 ' adenylation correlated with the reduced association between miRNAs and miRISC $[19,20]$. Regardless of how this controversy is ultimately resolved, both modifications can impact miRNA processing and activity profoundly.

Functions of the most abundantly tailed miR-8, bantam and miR-281 are not known yet in A. gambiae. It has been reported that bantam downregulates ecdysone signaling during larval development in Drosophila [46]. In addition, the ecdysone-responsive Drosophila miR-8 
regulates insulin signaling and innate immune homeostasis $[47,48]$. The comparison of consistently adenylated and uridylated miRNA sets identified in this study and reported in [34] revealed that the described 3'-end additions are not conserved in Drosophila homologous miRNAs. Nevertheless, the detected 3' extensions of miR-8 and bantam might be a signature of their activity in the complex regulatory network of insulin and ecdysone signaling in $A$. gambiae females triggered by blood meal intake.

The Anopheles genus separated approximately 120-190 milllion years ago from another blood-feeding mosquito genus Aedes, which is the main arbovirus vector responsible for transmitting alpha- and flaviviruses to humans. Despite the divergence and complexity of the Aedes genome with respect to Anopheles, the comparative analysis of our miRNA data sets and Ae. aegypti miRNAs revealed that more than a half of miRNAs were evolutionary conserved between these species. Overall, the relative arm usage of homologous miRNA loci was largely consistent in both Anopheles and Aedes. Yet, our study revealed an example of a remarkable change in the relative arm usage frequency in mir-1175 and mir-31 loci in response to blood feeding. One third of Anopheles miRNAs are closely linked in the genome, and such clustering organization is largely conserved between Aedes and Anopheles. Importantly, we described de novo emergence of species-specific miRNAs together with miRNA gene duplication and/or segregation in new clusters and further cluster fragmentation, which might be important for shaping of vector competence traits in these insects.

Functional arm switching significantly diversifies the regulatory capacity of miRNA genes. Selection of the preferred arm is regulated in a tissue- and organ-specific manner during development [20,32]. We did not detect dramatic changes in arm switching between sugar- and blood-fed Anopheles mosquitoes, whereas substantial fluctuations in the 5'/3'-arm usage were observed. Interestingly, we identified two examples of Anopheles miRNA loci, mir-305 and mir-1889 with absence of strong asymmetry in the preferred strand selection (Table 2). Furthermore, the preferred arm usage in mir-219 and mir-3840 loci was tilted after blood feeding to a nearly equal production of mature and star sequence species. All together, our extended set of Anopheles miRNAs and their isoforms provides a ground for further experimental studies of miRNA patterns and biological functions in A. gambiae.

Comparative analysis of miRNA abundance revealed dramatically elevated levels of miR-92a after blood meal intake. Interestingly, the sequence fraction assigned to miR-92a was enriched in mmu-miR-92a derived from the blood-host mouse. The mammalian miR-92a is a member of the conserved mir-17-92 cluster, whose over-expression is associated with lymphomas and other cancers [49]. Characterization of miRNA expression profiles in human blood revealed abundant expression of miR-92 in mature erythrocytes [50]. Consistent with this observation, elevated levels of human miR-92a were detected in the mosquito small RNA libraries prepared from females fed on human donor blood (not shown). We identified a set of highly abundant murine miRNAs in mosquito females fed on regular and infected blood (Additional file 3: Table S2). Interestingly, the $P$. berghei infection was associated with significant changes in abundance of mmu-mir-5105, mmu-mir-5115, mmu-mir-6243 and mmu-mir-5109. It has been reported that host bloodderived factors, such as human insulin, can modulate immunity and susceptibility of Anopheles mosquitoes to human Plasmodium infections [1]. However, the function of miRNAs derived from host-blood in mosquito physiology and anti-Plasmodium defenses has not been examined and requires further investigation.

It has been previously reported that Plasmodium infection was associated with significant changes in the expression of miR-34, miR-1174, miR-1175 and miR-989 detected at 24-48 h after P. berghei infection [25]. Interestingly, no overlap between sets of differentially expressed miRNAs reported in this study and by Winter et al. [25] was found. This discrepancy most probably reflects the dynamic changes in the miRNA expression profiles after an infectious meal. The further detailed study of the Plasmodium-responsive miRNA expression patterns and function may uncover new pathways and effectors that limit the parasite development within its insect host.

\section{Conclusions}

This study provides transcriptional evidences based on the 5p- and 3p-associated read abundance for $123 \mathrm{miR}$ NAs, including distinct 65 miRNAs previously reported in miRBase and 58 newly identified miRNAs in A. gambiae. Out of the newly described miRNAs, 21 novel miRNAs are potentially specific to A. gambiae. Importantly, sequence read abundance of certain miRNAs, such as newly identified mir-981, mir-33 and other miRNAs (Table 2) was not associated with the mature sequences predicted by sequence similarity. We extended a list of known mosquito-specific miRNAs previously reported by Li et al. [30] by describing 4 novel miRNAs conserved in Aedes (Table 1; Additional file 1: Figure S3). Detailed bioinformatics analysis provided evidences for functionally significant variations in mature sequences of Anopheles miRNAs and their isoforms occurring at their 5'- and 3 '-ends in sugar- and blood-fed mosquitoes (Figure 3). Moreover, we observed substantial variations in relative arm usage and arm-switching events showing the 
existence of species-specific production of dominant mature miRNAs induced by blood feeding in mosquitoes (Table 2, Figure 4). We identified new conserved and fragmented miRNA clusters and A. gambiae-specific miRNA gene duplication (Figure 5). Taken together, sequence variations, functional shifting and switching in mature miRNA sequences, described in this study, diversify significantly miRNA regulatory capacity in A. gambiae.

A. gambiae mosquitoes are the major vectors of human malaria in sub-Saharan Africa. We identified a set of the differentially expressed miRNAs that early respond to normal and infectious blood meals. The expression levels of the highly abundant miRNAs, miR-7 and exogenous mmu-miR-92a were significantly increased in blood-fed mosquitoes; while miR-317 and miR-2940 were significantly upregulated after $P$. berghei parasite infections. Further experimental study will require to discern the functions of the exogenous miRNAs derived from the host-blood and the Plasmodium-responsive miRNAs in the mosquito physiology and immunity.

\section{Methods}

Sample preparation and small RNA sequencing

A. gambiae G3 strain was reared and maintained in humidified chambers at $28^{\circ} \mathrm{C}$ with a $12 \mathrm{~h}$ light/dark cycle. For small RNA sequencing, 4-5 day old female mosquitoes were collected $3 \mathrm{~h}$ after a regular and an infectious blood feeding on the anaesthetized CD1 mice. Females fed on $10 \%$ sugar solution were used as a control. Infectious blood feeding was performed at $21^{\circ} \mathrm{C}$ on $\mathrm{CD} 1$ mice infected with the PbGFPCON strain [51] and fed mosquitoes were kept at $21^{\circ} \mathrm{C}$. Two independent biological replicates containing sugar-fed, blood-fed and P. berghei-infected females and a single replicate of Ago1- and Ago2-silenced females were used for small RNA cDNA library preparation. Total RNA was isolated from 30-50 females using a TRI Reagent (MRC). The strand-specific cDNA libraries with different barcodes (6 base index) were generated using a TruSeq Small RNA kit v2 (Illumina). The cDNA libraries were amplified by 13 cycles of polymerase chain reaction (PCR). The final 140-150 nt products were purified and sequenced in the Deep Sequencing facility of the IGBMC using the Illumina sequencing platform. The following adaptors and primers were used for cDNA synthesis and PCR amplification, 3' ligation adapter: 5'-pUCGUAUGCCGU CUUCUGCUUGUidT-3'; 5' ligation adapter: 5'-GUUCA GAGUUCUACAGUCCGACGAUC-3'; reverse transcription primer: 5'-CAAGCAGAAGACGGCATACGA-3'; PCR forward primer: 5'-CAAGCAGAAGACGGCATACGA3'; PCR reverse primer: 5'-AATGATACGGCGACCA CCGACAGGTTCAGAGTTCTACAGTCCGA-3'; sequencing primer: 5'-CGACAGGTTCAGAGTTCTACAGTCC GACGATC-3'.

\section{Data deposition}

Sequencing data have been deposited in the GEO database under the NCBI-GEO accession number GSE50396.

\section{miRNA identification and prediction}

Sequence reads were processed using the CASAVA1.8 pipeline (Illumina). Non-coding RNA profiling was performed by the ncPRO-seq analysis pipeline [52]. miRDeep2 analysis [53] was employed to detect potential miRNAs from raw RNA sequencing data using default parameters. To quantify miR-71 associated sequence reads the following algorithm developed in-house was used: sequence reads mapped to Agamp3 genome assembly, maximum 2 nt mismatches were allowed. Multiple position mapping was enabled and the weight of multiple mapping reads was considered. We allowed 2 nt upstream and downstream shifts in the mapping window for sequenced miR-71-5p and miR-71-3p, if they fall within the same positions on the predicted miRNA71 precursor. The thermodynamic stability of the secondary structures of the flanking genomic sequences was analyzed using RNAfold and Mfold [54,55]. IGV2.0 viewer was used to visualize sequence reads mapped to the reference genome. To identify putative homologs, sequence alignment of $A$. gambiae miRNAs with mature miRNAs was performed using Ae aegypti and $D$. melanogaster data sets (miRBase v19) and manually inspected. Mosquito miRNAs showing exact seed matches and sharing more than $70 \%$ of sequence similarity were considered as A. gambiae homologs of the corresponding known miRNAs. Anopheles miRNAs with a low level of sequence similarity were further used for the seed sequence alignment. The alignment was performed using the first $10 \mathrm{nt}$ at the 5'-end sequence of Anopheles miRNAs and sequences of known miRNA data sets, including Ae. aegypti (AaegL1), B. mori (SILKDB2.0), D. melanogaster (BDGP5.0), T. castaneum (Tcas3.0) and H. sapiens (GRCh37.p5). Multiple RNA sequence alignment was performed using the MARNA RNA tool. For quantification of miRNA expression levels, sequence reads with non-template additions at the 3'-end were included in the counts. The DESeq2.6 package was used to quantify and to assess miRNA differential expression, which was considered as significant at $p<0.05$. The raw log value of all single miRNA reads and the $5 \mathrm{p}$ - and 3p-derived reads are listed in Additional file 2: Table S1 and in Additional file 1: Figures S1-2, respectively. Detected $M$. musculus miRNAs in small RNA cDNA libraries are listed in Additional file 3: Table S2. Small RNA expression in Ago-silenced mosquitoes was analyzed using miRBase v19, miRDeep2 and Rfam databases. The raw and normalized read frequencies of small RNAs are listed in Additional file 4: Table S3. 


\section{Plasmid construction and RNA-based silencing}

RNA interference was used to silence Ago1 and Ago2 expression in adult female mosquitoes. Ago1 (AGAP011717): XhoI-XbaI 432 bp PCR-amplified fragment was subcloned from the 20AA09 clone of the Gateway (Invitrogen) immune library described in [56] into the pLL110 vector carrying two T7 promoters. Ago2 (AGAP011537): StuI-XhoI 500 bp fragment was PCR-amplified from a cDNA (A. gambiae G3 strain) and cloned into pLL110. The following PCR primers were used, Ago1 forward primer: 5'-CTGCACCGTTACAGACACG-3'; Ago1 reverse primer: 5'CCAAGTTGCCCCATCCC-3; Ago2 forward primer: 5'-aaaAGGCCTGCCACCGGTAGTGCC-3'; Ago2 reverse primer: 5'-ccgctcgagGTTTTCAGCACGCCCAA $A T C-3$ '. Sense and anti-sense single-stranded RNAs were synthesized using MEGAscript T7 kit (Ambion), purified using MEGAclear kit (Ambion) and annealed in an equimolar ratio. One-day post-emerged $\mathrm{CO}_{2}$-anaesthetized mosquito females were injected intrathoracically with $0.6 \mu \mathrm{g}$ of dsRNA using nano-injector (Nanoject II, Drummond). Efficacy of RNA silencing on gene expression was analyzed $24 \mathrm{~h}$ after dsRNA injection by quantitative real-time PCR (qRT-PCR).

\section{qRT-PCR}

Efficacy of Ago1 and Ago2 silencing in the RNA samples used for RNA sequencing was assessed by SYBR Greenbased qPCR (ABI). cDNAs were sythesized from $1 \mu \mathrm{g}$ of total RNA samples using random primers and RevertAid H Minus cDNA synthesis kit (Fermentas). Ribosomal protein L19 gene (RplL19) was used as an internal control to normalize Ago1 and Ago2 gene expression. Specific primers were designed using Primer Express 3.0 (ABI): Ago1 forward primer 5'-ACGATGCGGCGC AAGTAT-3'; Ago1 reverse primer 5'-CGGGAAGGA TTGCATTTGTG-3'; Ago2 forward primer 5'-ATGCTC AAGATCAACGCCAAA-3'; Ago2 reverse primer 5'-TG AGCGGGTGCGTAACGT-3'; RpL19 forward primer 5'-CCAACTCGCGACAAAACATTC-3'; RpL19 reverse primer 5'- ACCGGCTTCTTGATGATCAGA-3'. TaqMan qRT-PCR based quantification of miR-989 and $5.8 \mathrm{~S}$ rRNA expression levels were performed using TaqMan miRNA RT kit and custom TaqMan small RNA assays (ABI). Relative miR-989 levels were normalized to $5.8 \mathrm{~S}$ rRNA. $10 \mathrm{ng}$ of total RNA were used for cDNA synthesis. The RT reaction without reverse transcriptase was used as a negative control. PCR reactions were performed on an OneStep Plus thermocycler (ABI), according to the manufacturer's protocol, and each measurement was derived from three independent biological replicates. Relative quantification of gene expression was performed using the comparative $\mathrm{Ct}(\Delta \Delta \mathrm{Ct})$ method.

\section{miRNA sequence variations and preferred arm usage}

Analyses of the miRNA sequence variations and the relative arm usage were done using an algorithm developed in-house. The quantification was executed with a pipeline of custom developed Python scripts available upon request. The most abundant sequence reads were used as a reference. No mismatch was allowed within mature miRNA sequences. To identify template-directed variations, sequence reads with $3 \mathrm{nt}$ and less difference at the 5'-and 3'-ends from the most frequently sequenced reads derived from both arms were retrieved and quantified. To characterize non-template directed nucleotide additions, sequence reads with single nucleotide addition mismatched at the 3'-end were quantified. Sequence compilations for specific mature miRNAs were generated using WebLogo3.3. The 5'- and 3'-arm usage of miRNA hairpins was quantified as a proportion of the $5 \mathrm{p}$-associated reads with respect to the total number of reads from miRNAs in A. gambiae. Published small RNA data sets for D. melanogaster [34] and Ae. aegypti female gutspecific libraries [30] were used for the relative arm usage analyses. The relative arm usage was calculated as a ratio of the 5'- to the 3'-arm associated reads of the hairpin precursor and shown as log values. To identify A. gambiae miRNA clusters, a $1 \mathrm{~kb}, 5 \mathrm{~kb}, 10 \mathrm{~kb}$ and $50 \mathrm{~kb}$ cut-off were used. Cluster conservation analyses between A. gambiae and Ae. aegypti was performed using $10 \mathrm{~kb}$ cut-off for genomic distance.

\section{Ethics statement}

All vertebrate animals were housed and handled in accordance with the animal protection law (\$8 Tierschutzgesetz) and both institutional (Max Planck Society) and national (Landesamt für Gesundheit und Soziales (LAGeSo) regulations. All experimental procedures on mice were approved by the committee for animal use and protection (LAGeSo permit number: $\mathrm{H}$ 0027/12).

\section{Additional files}

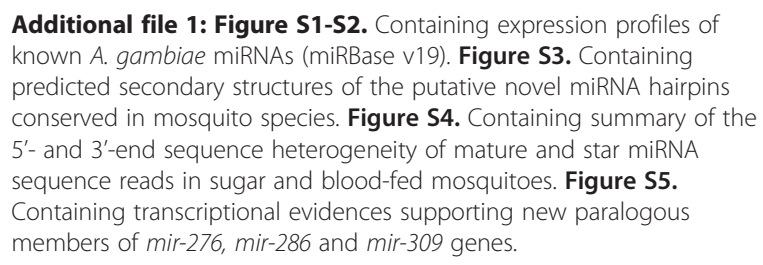

Additional file 2: Table S1. Containing A. gambiae miRNA counts from small RNA sequencing of sugar-fed, blood-fed and $P$. berghei infected mosquitoes.

Additional file 3: Table S2. Containing M. musculus miRNA counts detected in mosquito small RNA cDNA libraries.

Additional file 4: Table S3. Containing quantification of the $A$. gambiae small RNA expression in Ago-silenced mosquitoes. 


\section{Competing interests}

The authors declare that they have no competing interests.

\section{Authors' contributions}

IB and EL designed the project, and EL revised the manuscript. TYe performed bioinformatics analysis. IB conducted experimental work, analysed and interpreted data, oversaw the bioinformatics analysis and wrote the manuscript. All authors read and approved the final manuscript.

\section{Acknowledgement}

We would like to acknowledge Julien Soichot and Olivier Pichon (IBMC); Dana Tschierske and Hanne Krueger (MPIIB) for maintaining mosquito colonies and technical assistance with mouse blood-feeding. We thank Bernard Jost, Muriel Koch, Géraldine Bamba and Serge Vicaire (IGBMC) for the assistance with small RNA libraries preparation and high-throughput sequencing. We thank Alexandra Krupa and John R. Clayton (IBMC) for helpful discussion, for reading of this manuscript and providing critical comments. This work was supported by the European Community's Seventh Framework Programme (FP7/2007-2013) under grant agreements no. 242095-EVIMalar and no. 223601-MALVECBLOK.

\section{Author details}

'Department of Vector Biology, Max Planck Institute for Infection Biology, Berlin 10117, Germany. 'Microarrays and deep sequencing platform, IGBMC, Illkirch, Cedex 67404, France.

Received: 7 November 2013 Accepted: 25 June 2014

Published: 4 July 2014

\section{References}

1. Pakpour N, Corby-Harris V, Green G, Smithers H, Cheung K, Riehle M, Luckhart S: Ingested human insulin inhibits the mosquito NF-kappaBdependent immune response to Plasmodium falciparum. Infect Immun 2012, 80:2141-2149.

2. Marois $\mathrm{E}$ : The multifaceted mosquito anti-Plasmodium response. Curr Opin Microbiol 2011, 14:429-435.

3. Bartel D: MicroRNAs: genomics, biogenesis, mechanism, and function. Cell 2004, 116:281-297.

4. Smibert P, Lai E: A view from Drosophila: multiple biological functions for individual microRNAs. Semin Cell Dev Biol 2010, 21:745-753.

5. Kim V, Han J, Siomi M: Biogenesis of small RNAs in animals. Nat Rev Mol Cell Biol 2009, 10:126-139.

6. Okamura K, Liu N, Lai E: Distinct mechanisms for microRNA strand selection by Drosophila Argonautes. Mol Cell 2009, 36:431-444.

7. Yang J-S, Lai E: Alternative miRNA biogenesis pathways and the interpretation of core miRNA pathway mutants. Mol Cell 2011, 43:892-903.

8. Diederichs $S$, Haber D: Dual role for argonautes in microRNA processing and posttranscriptional regulation of microRNA expression. Cell 2007, 131:1097-1108.

9. Zhou R, Hotta I, Denli A, Hong P, Perrimon N, Hannon G: Comparative analysis of argonaute-dependent small RNA pathways in Drosophila. Mol Cell 2008, 32:592-599.

10. Campbell C, Black W, Hess A, Foy B: Comparative genomics of small RNA regulatory pathway components in vector mosquitoes. BMC Genomics 2008, 9:425.

11. Hoa N, Keene K, Olson K, Zheng L: Characterization of RNA interference in an Anopheles gambiae cell line. Insect Biochem Mol Biol 2003, 33:949-957.

12. Bartel D: MicroRNAs: target recognition and regulatory functions. Cell 2009, 136:215-233.

13. Fabian $\mathrm{M}$, Sonenberg $\mathrm{N}$ : The mechanics of miRNA-mediated gene silencing: a look under the hood of miRISC. Nat Struct Mol Biol 2012, 19:586-593.

14. Wienholds E, Kloosterman W, Miska E, Alvarez-Saavedra E, Berezikov E, de Bruijn E, Horvitz H, Kauppinen S, Plasterk R: MicroRNA expression in zebrafish embryonic development. Science (New York, NY) 2005, 309:310-311.

15. Stark A, Brennecke J, Bushati N, Russell R, Cohen S: Animal MicroRNAs confer robustness to gene expression and have a significant impact on 3'UTR evolution. Cell 2005, 123:1133-1146.

16. Kim Y-K, Heo I, Kim V: Modifications of small RNAs and their associated proteins. Cell 2010, 143:703-709.
17. Berezikov E: Evolution of microRNA diversity and regulation in animals. Nat Rev Genet 2011, 12:846-860.

18. Katoh T, Sakaguchi Y, Miyauchi K, Suzuki T, Kashiwabara S-I, Baba T, Suzuki T: Selective stabilization of mammalian microRNAs by 3 ' adenylation mediated by the cytoplasmic poly(A) polymerase GLD-2. Genes Dev 2009, 23:433-438.

19. Burroughs $A$, Ando $Y$, de Hoon M, Tomaru $Y$, Nishibu T, Ukekawa $R$, Funakoshi T, Kurokawa T, Suzuki H, Hayashizaki Y, Daub C: A comprehensive survey of $3^{\prime}$ animal miRNA modification events and a possible role for $3^{\prime}$ adenylation in modulating miRNA targeting effectiveness. Genome Res 2010, 20:1398-1410.

20. Zhou H, Arcila M, Li Z, Lee E, Henzler C, Liu J, Rana T, Kosik K: Deep annotation of mouse iso-miR and iso-moR variation. Nucleic Acids Res 2012, 40:5864-5875.

21. Heo I, Joo C, Kim Y-K, Ha M, Yoon M-J, Cho J, Yeom K-H, Han J, Kim V: TUT4 in concert with Lin28 suppresses microRNA biogenesis through pre-microRNA uridylation. Cell 2009, 138:696-708.

22. Eulalio A, Schulte L, Vogel J: The mammalian microRNA response to bacterial infections. RNA Biol 2012, 9:742-750.

23. Cullen B: MicroRNAs as mediators of viral evasion of the immune system. Nat Immunol 2013, 14:205-210.

24. Hakimi MA, Cannella D: Apicomplexan parasites and subversion of the host cell microRNA pathway. Trends Parasitol 2011, 27:481-486.

25. Winter F, Edaye S, Huttenhofer A, Brunel C: Anopheles gambiae miRNAs as actors of defence reaction against Plasmodium invasion. Nucleic Acids Res 2007, 35:6953-6962.

26. Mead E, Li M, Tu Z, Zhu J: Translational regulation of Anopheles gambiae mRNAs in the midgut during Plasmodium falciparum infection. BMC Genomics 2012, 13:366.

27. Wang X, Zhang J, Li F, Gu J, He T, Zhang X, Li Y: MicroRNA identification based on sequence and structure alignment. Bioinformatics 2005 , 21:3610-3614

28. Chatterjee $R$, Chaudhuri $K$ : An approach for the identification of microRNA with an application to Anopheles gambiae. Acta Biochim Pol 2006 53:303-309

29. Mead E, Tu Z: Cloning, characterization, and expression of microRNAs from the Asian malaria mosquito, Anopheles stephensi. BMC Genomics 2008, 9:244.

30. Li S, Mead E, Liang S, Tu Z: Direct sequencing and expression analysis of a large number of miRNAs in Aedes aegypti and a multi-species survey of novel mosquito miRNAs. BMC Genomics 2009, 10:581.

31. Skalsky R, Vanlandingham D, Scholle F, Higgs $S$, Cullen B: Identification of microRNAs expressed in two mosquito vectors, Aedes albopictus and Culex quinquefasciatus. BMC Genomics 2010, 11:119.

32. Chiang $H$, Schoenfeld L, Ruby J, Auyeung V, Spies N, Baek D, Johnston W, Russ C, Luo S, Babiarz J, Blelloch R, Schroth G, Nusbaum C, Bartel D: Mammalian microRNAs: experimental evaluation of novel and previously annotated genes. Genes Dev 2010, 24:992-1009.

33. Kozomara A, Griffiths-Jones S: miRBase: integrating microRNA annotation and deep-sequencing data. Nucleic Acids Res 2011, 39:7.

34. Berezikov E, Robine N, Samsonova A, Westholm J, Naqvi A, Hung J-H, Okamura K, Dai Q, Bortolamiol-Becet D, Martin R, Zhao Y, Zamore P, Hannon G, Marra M, Weng Z, Perrimon P, Lai E: Deep annotation of Drosophila melanogaster microRNAs yields insights into their processing, modification, and emergence. Genome Res 2011, 21:203-215.

35. Marco A, Hui J, Ronshaugen M, Griffiths-Jones S: Functional shifts in insect microRNA evolution. Genome Biol Evol 2010, 2:686-696.

36. Landgraf $P$, Rusu M, Sheridan R, Sewer A, lovino N, Aravin A, Pfeffer S, Rice A, Kamphorst A, Landthaler M, Lin C, Socci N, Hermida L, Fulci V, Chiaretti S, Foa R, Schliwka J, Fuchs U, Novosel A, Muller R-U, Schermer B, Bissels U, Inman J, Phan Q, Chien M, Weir D, Choksi R, De Vita G, Frezzetti D, Trompeter $\mathrm{H}-\mathrm{I}$, et al: A mammalian microRNA expression atlas based on small RNA library sequencing. Cell 2007, 129:1401-1414.

37. Ruby J, Stark A, Johnston W, Kellis M, Bartel D, Lai E: Evolution, biogenesis, expression, and target predictions of a substantially expanded set of Drosophila microRNAs. Genome Res 2007, 17:1850-1864

38. Morin R, O'Connor M, Griffith M, Kuchenbauer F, Delaney A, Prabhu A-L, Zhao Y, McDonald H, Zeng T, Hirst M, Eaves C, Marra M: Application of massively parallel sequencing to microRNA profiling and discovery in human embryonic stem cells. Genome Res 2008, 18:610-621. 
39. Seitz $H$, Ghildiyal M, Zamore P: Argonaute loading improves the $5^{\prime}$ precision of both MicroRNAs and their miRNA* strands in flies. Curr Biol 2008, 18:147-151.

40. Fernandez-Valverde $S$, Taft $R$, Mattick J: Dynamic isomiR regulation in Drosophila development. RNA 2010, 16:1881-1888.

41. Wei C, Salichos L, Wittgrove C, Rokas A, Patton J: Transcriptome-wide analysis of small RNA expression in early zebrafish development. RNA 2012, 18:915-929.

42. Liu N, Okamura K, Tyler D, Phillips M, Chung W-J, Lai E: The evolution and functional diversification of animal microRNA genes. Cell Res 2008, 18:985-996.

43. Okamura K, Phillips M, Tyler D, Duan H, Chou Y-t, Lai E: The regulatory activity of microRNA* species has substantial influence on microRNA and 3' UTR evolution. Nat Struct Mol Biol 2008, 15:354-363.

44. Fukunaga $\mathrm{R}$, Han B, Hung J-H, Xu J, Weng Z, Zamore P: Dicer partner proteins tune the length of mature miRNAs in flies and mammals. Cell 2012, 151:533-546.

45. D'Ambrogio A, Gu W, Udagawa T, Mello C, Richter J: Specific miRNA stabilization by Gld2-catalyzed monoadenylation. Cell Rep 2012, 2:1537-1545.

46. Boulan L, Martin D, Milan M: bantam miRNA promotes systemic growth by connecting insulin signaling and ecdysone production. Curr Biol 2013, 23:473-478.

47. Jin H, Kim V, Hyun S: Conserved microRNA miR-8 controls body size in response to steroid signaling in Drosophila. Genes Dev 2012, 26:1427-1432.

48. Choi I, Hyun S: Conserved microRNA miR-8 in fat body regulates innate immune homeostasis in Drosophila. Dev Comp Immunol 2012, 37:50-54.

49. Xiao C, Srinivasan L, Calado D, Patterson H, Zhang B, Wang J, Henderson J, Kutok J, Rajewsky K: Lymphoproliferative disease and autoimmunity in mice with increased miR-17-92 expression in lymphocytes. Nat Immuno 2008, 9:405-414

50. Chen S-Y, Wang Y, Telen M, Chi J-T: The genomic analysis of erythrocyte microRNA expression in sickle cell diseases. PLOS ONE 2008, 3(6):e2360.

51. Franke-Fayard B, Trueman H, Ramesar J, Mendoza J, van der Keur M, van der Linden $\mathrm{R}$, Sinden $\mathrm{R}$, Waters $A$, Janse C: A Plasmodium berghei reference line that constitutively expresses GFP at a high level throughout the complete life cycle. Mol Biochem Parasitol 2004, 137:23-33.

52. Chen C-J, Servant N, Toedling J, Sarazin A, Marchais A, Duvernois-Berthet E, Cognat V, Colot V, Voinnet O, Heard E, Ciaudo C, Barillot E: ncPRO-seq: a tool for annotation and profiling of ncRNAs in sRNA-seq data. Bioinformatics 2012, 28:3147-3149.

53. Friedlander M, Mackowiak S, Li N, Chen W, Rajewsky N: miRDeep2 accurately identifies known and hundreds of novel microRNA genes in seven animal clades. Nucleic Acids Res 2012, 40:37-52.

54. Hofacker I: Vienna RNA secondary structure server. Nucleic Acids Res 2003 31:3429-3431

55. Zucker M: Mfold web server for nucleic acid folding and hybridization prediction. Nucleic Acids Res 2003, 31:3406-3415

56. Frolet C, Thoma M, Blandin S, Hoffmann J, Levashina E: Boosting NF-kappaB-dependent basal immunity of Anopheles gambiae aborts development of Plasmodium berghei. Immunity 2006, 25:677-685.

doi:10.1186/1471-2164-15-557

Cite this article as: Biryukova et al:: Transcriptome-wide analysis of microRNA expression in the malaria mosquito Anopheles gambiae. BMC Genomics 2014 15:557.

\section{Submit your next manuscript to BioMed Central and take full advantage of:}

- Convenient online submission

- Thorough peer review

- No space constraints or color figure charges

- Immediate publication on acceptance

- Inclusion in PubMed, CAS, Scopus and Google Scholar

- Research which is freely available for redistribution 